\title{
Offshore Wind and Wave Energy Assessment around Malè and Magoodhoo Island (Maldives)
}

\author{
Pasquale Contestabile ${ }^{1}$, Enrico Di Lauro ${ }^{1}$, Paolo Galli ${ }^{2,3,4}$, Cesare Corselli ${ }^{2,5}$ and \\ Diego Vicinanza $1,2,6, *$ \\ 1 Department of Civil Engineering, Design, Building and Environment, Università degli studi della \\ Campania "Luigi Vanvitelli", Via Roma 29, 81031 Aversa (Caserta), Italy; \\ pasquale.contestabile@unicampania.it (P.C.); enrico.dilauro@unicampania.it (E.D.L.) \\ 2 CONISMA-National Inter-University Consortium of Marine Sciences, Piazzale Flaminio 9, \\ 00196 Roma, Italy \\ 3 Department of Biotechnology and Biosciences, University of Milano-Bicocca, Piazza della Scienza 2, \\ 20126 Milan, Italy; paolo.galli@unimib.it \\ 4 MaRHE Center (Marine Research and High Education Center), Magoodhoo Island, \\ 20217 Faafu Atoll, Maldives \\ 5 Department of Earth and Environmental Sciences, University of Milano-Bicocca, \\ Piazza della Scienza 1 and 4, 20126 Milan, Italy; cesare.corselli@unimib.it \\ 6 Stazione Zoologica Anton Dohrn, Villa Comunale, 80121 Napoli, Italy \\ * Correspondence: diego.vicinanza@unicampania.it; Tel.: +39-081-50-10-245 \\ Academic Editor: Marc A. Rosen \\ Received: 3 March 2017; Accepted: 11 April 2017; Published: 14 April 2017
}

\begin{abstract}
The Maldives are situated in the remote equatorial Indian Ocean, covering $900 \mathrm{~km}$ from north to south. The 26 coral atolls forming the archipelago are composed of sand and coral with a maximum height of about $2.30 \mathrm{~m}$ above the mean sea level. Periodic flooding from storm surges and the frequent freshwater scarcity are perceived by the population and the economic operators as the major environmental stresses. Moreover, the strong dependence on imported fossil fuels increases, even more, the environmental concerns. Diesel, in fact, still represents the main source of power generation, typically through privately managed small diesel sets. The real challenge for this area is to promote the environmental quality with socioeconomic growth. The present study aims to evaluate the strategic effectiveness to face these issues by wave and offshore wind energy. Resources using a 10-year hindcast dataset are here examined. The annual offshore wave power was found to range between $8.46 \mathrm{~kW} / \mathrm{m}$ and $12.75 \mathrm{~kW} / \mathrm{m}$, while the $10 \mathrm{~m}$ and $100 \mathrm{~m}$ mean wind power density is respectively $0.08 \mathrm{~kW} / \mathrm{m}^{2}$ and $0.16 \mathrm{~kW} / \mathrm{m}^{2}$. Based on these results, an environmentally and socio-economically sustainable best-case scenario is constructed and two atoll islands (Malè and Magoodhoo) are specifically investigated. As a result, multifunctional structures and multi-use systems, which combine power generation, desalinization and coastal defence, are strongly recommended.
\end{abstract}

Keywords: wave energy resource; offshore wind resource; Maldives; multi-use systems

\section{Introduction}

The Maldives, an island nation in the Indian Ocean, are largely recognized as one of the most beautiful countries in the world and an example of a natural paradise. However, a paradise with a particular issue: it is one of the most exposed countries in the world to the volatility and the rising of oil prices and it is extremely vulnerable to the effects of the climate change. The reason is twofold: country's strong dependency on fossil fuel, which is essentially the only exploitable source of energy, and the natural conformation of the Archipelago. The Maldives are composed exclusively of corals 
and sand, without surface bodies of fresh waters and with no point higher than $2.3 \mathrm{~m}$ above mean sea level. Moreover, much of the groundwater is polluted and cannot be used, which makes the Maldives an easy prey of emergency situations and speculations [1].

Several recent studies indicate a profound reorganization of the energy market and environmental policy in the Maldives, aiming to convert the island nation as the world's first carbon neutral country. Solar photovoltaic, wind energy and biomass represent the key renewable resources according to the Scaling up Renewable Energy Program (SREP) under the Climate Investment Funds [2]. Energy from waste is under consideration. As for tidal and marine currents, a study conducted by the Robert Gordon University of Scotland in 2011 concluded that current technologies are not yet relevant [3]. However, the SREP investment plan does not include in its study neither onshore/offshore wind source nor wave energy. Recently, the Government of the Maldives has recognized the need to improve the information available on solar and wind potential as it requested the support of the World Bank and ESMAP's Renewable Energy Mapping Initiative to help carry out a resource assessment and mapping [4], showing some preliminary results. With respect to traditional wind resource, offshore wind farms present benefits that include (a) lower visual and noise impact through distance from the shoreline and (b) higher wind resource (larger wind velocities) with lower turbulence levels than adjacent land sites. Furthermore, since industry is seeking economically viable devices for commercial deployment, the economic feasibility and regularity in the power output of offshore wind farms could be enhanced combining two forms of energy production, i.e., wind and wave, in one platform. Cases of integration of wave and wind power have been studied by [5-23], demonstrating as the joint exploitation of offshore wind and wave energy resources can have a number of advantages, including:

- $\quad$ higher quality of produced power when mixing the power from wind and wave energy;

- $\quad$ higher regularity of power delivered to the grid when swells continue after the wind declines;

- reducing risk and cost through sharing components, infrastructure, submarine electric cables and maintenance activities;

- less area and environmental impact for combined farms through the sharing of space.

Over the last two decades several efforts have been made to map the offshore wind and wave energy resource. Maps of the global offshore wind energy resources have earlier been published in [24], and wind resource assessment was analysed by, e.g., [25-42].

Considerable works have been undertaken on wave energy assessment in several areas worldwide (e.g., [43-67]). A global synthesis has been published in the review book by Cruz (2008), based on the WorldWaves data.

The present work analyses the blue energy resources along the Maldives coastline, using a 10-years data series provided by the European Centre for Medium-Range Weather Forecasts (ECMWF) dataset [68]. The spatial distribution of both energy sources is analysed using data from 10 points along the coast. The approach used in the present study follows a widely validated methodology in literature for both the wind and the wave energy assessment undertaken for several locations worldwide [25-67], but the two assessments have been concurrently compared and innovative applications of hybrid technologies have been considered.

In particular, the objectives of this work are:

1. to characterize the wave climate around the study area, emphasizing wave power assessment and the involvement of different sea states (e.g., monthly variability and long period swell influence);

2. to provide a comprehensive study of offshore wind resource, provided at a height of $10,25,55,80$ and $100 \mathrm{~m}$ and referring to three practical utility-scale wind turbines;

3. to draw a preliminary discussion about the realistic perspectives of blue farm installations around the Maldives coastline, also through practical case studies of these innovative technologies.

For the last point, a special attention has been paid to enhance the environmental and socio-economical sustainability of blue energy technologies, identifying the possible "cost saving 
synergies" between wave energy and other sea uses. Two case scenarios will be presented to illustrate the approach: both of them include a hybrid platform of wave and wind and another (non-energy) sea use. Two representative islands are analyzed:

- Malé, the capital, one of the most densely populated cities in the world, with approximately 23,000 people per $\mathrm{km}^{2}$ and an electricity demand of over $220 \mathrm{GWh} /$ year in 2012 [69];

- Magoodhoo, a remote small island on the Faafu Atoll, at a distance of $134 \mathrm{~km}$ from the capital, with a population of 683 inhabitants distributed on a surface of less than $0.36 \mathrm{~km}^{2}$.

The paper is structured as follows. Section 2 briefly describes the study area, presents the available data and introduces the methodology. In Section 3 the offshore wind energy and the wave energy resource along the Maldivian coasts are assessed. In Section 4 the results are discussed and the best-case scenarios for the two sites are specifically investigated, showing the combined maritime uses that can be more feasible. Conclusions are finally drawn in Section 5.

\section{Materials and Methods}

\subsection{Wave and Wind Data for the Study Area}

Since suitable buoy data and long-term historical wind record are not available off the coasts of the Maldives, the present work has been based on ERA-Interim dataset, freely available for downloading at the website (http://www.ecmwf.int/). This is a global atmospheric reanalysis provided by the European Centre for Medium-Range Weather (ECMWF), continuously updated in real time providing data from 1979. These operational numerical weather predictions are used to predict meteorological states, based on how the climate system evolves with time from an initial state. The ECMWF dataset is composed of a coupled ocean-atmosphere general circulation model, i.e., an atmospheric reanalysis coupled with a wave model integration where no wave parameters were assimilated, making the wave part a hindcast run. The Indian Ocean is covered by the base model grid with a resolution of $0.75^{\circ} \times 0.75^{\circ}$. Ten grid points have been selected in this study in order to cover the east and west side of the study area, covering a latitude from $1.5^{\circ} \mathrm{N}$ to $4.5^{\circ} \mathrm{N}$ (Figure 1). The five East grid points (on longitude $74.25^{\circ} \mathrm{E}$ ) are here termed E1-E5, while W1-W5 (on longitude $72^{\circ} \mathrm{E}$ ) are the West ones. These hindcast data provided by the ECMWF have been chosen for several reasons:

- ERA-Interim products are freely available on the ECMWF Data Server;

- due to the re-analysis nature, this dataset is free from a lack in the time series and the data should be considered as pre-processed from an error handling phase;

- for wave energy assessing purposes, this hindcast has been shown to be slightly conservative.

This conservative nature can be addressed to the peak attenuation due to the small sampling frequency (i.e., smaller than that of the traditional wave buoys). Moreover, in the characteristic meteorological spectrum of the model, the energy falls fairly rudely at about 125 miles, a dimension comparable to the model grid resolution. This approach makes results here presented reliable from an engineering point of view, albeit data validation is not possible since only limited measured wave time series are available for the analysed area. The main wind/wave parameters, such as the significant wave height $\left(H_{s}\right)$, the mean wave period $\left(T_{m}\right)$, mean wave direction $\left(\theta_{m}\right)$, the longitudinal component of $10 \mathrm{~m}$ wind speed $(u)$ and a latitudinal component of $10 \mathrm{~m}$ wind speed $(v)$ ranging from January / 2005 to December/2014, were extracted from the ERA-Interim archive. For the wave energy computation, all the ten ECMWF points have been used. Because of the great depth around the archipelago, with rapid change in bathymetry just few tens of meters from the coral reef, effect of refraction along the exposed coast of the Archipelago can be considered negligible. Therefore, offshore energy density assessment could be considered valid also for nearshore conditions. 


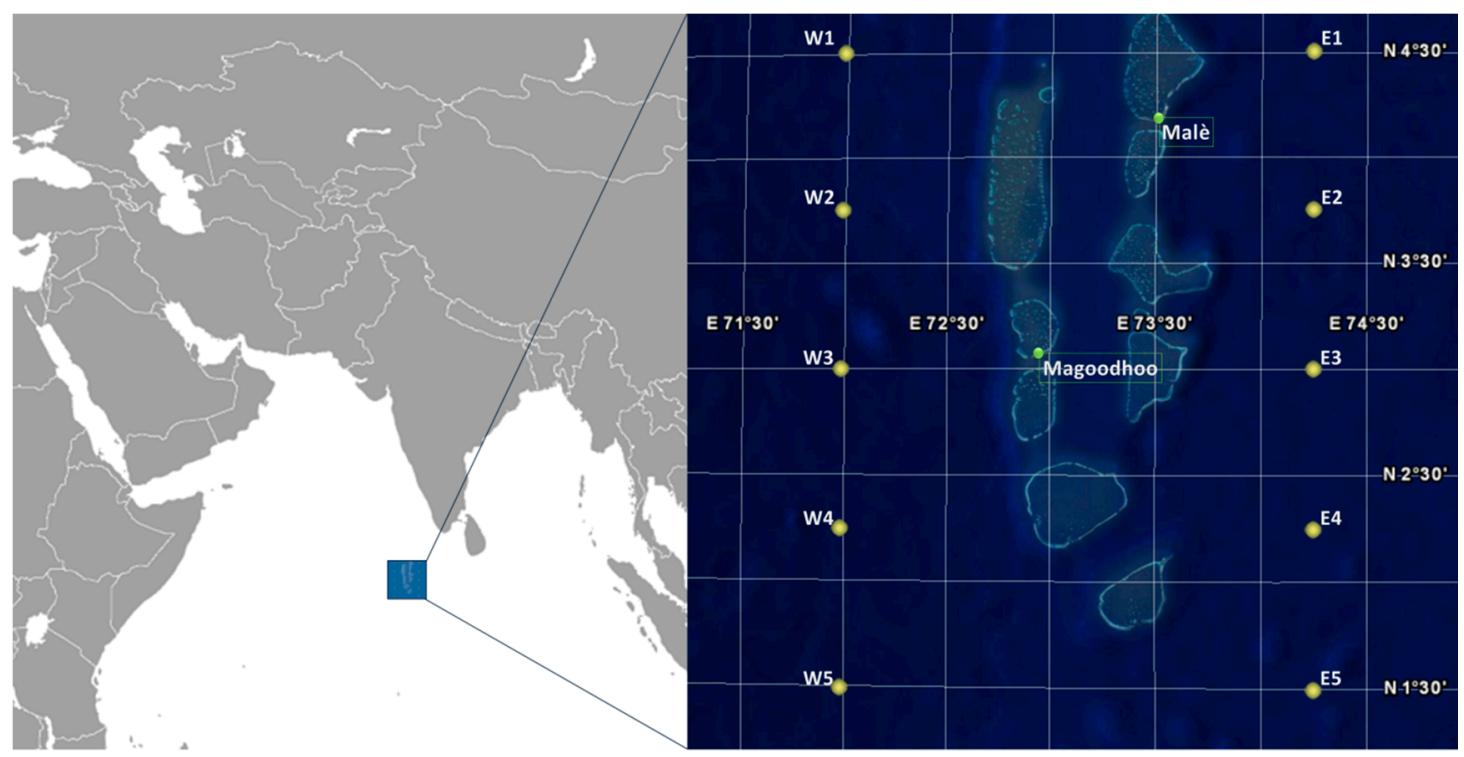

Figure 1. Map of Western Indian Ocean showing the location of ECMWF grid study points around the Maldives islands and the two focus sites (Malè ad Magoodhoo).

\subsection{Method for Wave Energy Potential Computation}

The methodology used to determine the offshore wave power follows that successfully applied to others deep waters regions (e.g., $[60,65]$. Regarding the calculation of the wave energy flux transmitted per unit width of waves, it is worth remarking that all reference points used in the present analysis are located in deep waters. For regular waves, the sum of kinetic and potential energy density per unit width can be computed according to the following well-known relationship:

$$
E_{\text {wave }}=\rho g H^{2} / 8
$$

where $\rho$ is the sea water density, $g$ is the gravity acceleration and $H$ is the wave height. It this work an average value of $\rho=1025 \mathrm{Kg} / \mathrm{m}^{3}$ has been adopted. The energy flux across a vertical section of unit width perpendicular to the wave propagation direction can be expressed as follows:

$$
P_{\text {wave }}=E_{\text {wave }} \times C_{g}
$$

in which $C_{g}$ is the group velocity. In case of deep water, $C_{g}$ can be expressed as follows:

$$
C_{g}=\frac{g}{2 \omega}
$$

where the wave frequency, $\omega$, is $2 \pi T$, being $T$ the wave period.

In a real sea state, the power of irregular waves can be described by a superposition of an infinite number of regular waves with different amplitudes and frequencies. Therefore, the wave energy flux using spectral parameters can be defined as:

$$
P=\rho g \int_{0}^{2 \pi} \int_{0}^{\infty} C_{g}(f, h) \times S(f, \theta) d f d \theta
$$

in which $S(f, \theta)$ denotes the directional wave spectrum and $C_{g}(f, h)$ denotes the general expression of the wave group velocity, expressed as:

$$
C_{g}(f, h)=\frac{1}{2}\left[1+\frac{2 k h}{\sinh (s k h)}\right] \sqrt{\frac{g}{h} \tanh (k h)}
$$


Wave height computation is based on the zero-order moment of the spectral function and readily estimated as:

$$
H_{m 0}=4 \sqrt{m_{0}}
$$

As a period parameter, the present method uses the energy period, $T_{e}$, defined as the ratio between the minus-one and the zeroth spectral moments:

$$
T_{e}=\frac{m_{-1}}{m_{0}}
$$

Being known the mean period $T_{m}$ from the hindcast dataset, it was assumed that $T_{e}=1.14 T_{m}$, according to the approach used in preparing the Atlas of UK Marine Renewable Energy Resources [70]. Hence, the wave power, under a wave crest 1 meter wide is given by:

$$
P_{\text {wave }}=\frac{\rho g^{2} H_{m 0}^{2} T_{e}}{64 \pi}
$$

For each six-hours triple $\left(H_{s}, T_{m}, \theta_{m}\right)$ provided at each hindcast point, the related power series using Equation (8) was computed. It is important to underline that the presence of atolls creates sheltered areas in their lee into which wave energy penetrates mainly through diffraction. Due to the high environmental value of the coral reef and internal waters of the archipelago, hypothesis of blue energy installations in the sheltered areas has not considered in this study. Therefore, analysis of wave energy density in the internal waters (therefore taking into account refraction, diffraction, etc.) is out of the scope of the present paper.

\subsection{Method for Wind Energy Potential Computation}

With regard to offshore wind power, the approach applied in this work follows previous studies (e.g., [6,40]). As known, the wind energy in an open air stream is proportional to the third power of the wind speed. In particular, the total amount of kinetic energy flowing through an area $R$ during the time $t$ is expressed as:

$$
E_{\text {wind }}=\frac{1}{2} \rho_{a} t R w^{3}
$$

where $\rho_{a}$ is the air density (a constant value of $\rho_{a}=1.177 \mathrm{Kg} / \mathrm{m}^{3}$ was adopted in this study considering an average annual air temperature of $27^{\circ}$ ) and $w$ is the wind speed computed from the horizontal North-East wind components $\left(\sqrt{u^{2}+v^{2}}\right)$. The eastward $(u)$ and the northward $(v)$ wind components provided by the ERA-Interim refer to the wind at $10-\mathrm{m}$ elevation above the mean sea surface. To calculate the actual wind speed at different heights, the logarithmic wind profile is used [71]:

$$
U(z)=H(H) \times\left(1+\frac{\ln \left(\frac{z}{H}\right)}{\ln \left(\frac{H}{z_{0}}\right)}\right)
$$

where $z$ is the general height above sea level, $H$ is the reference height of $10 \mathrm{~m}$, and $z_{0}$ is the terrain roughness parameter. The latter coefficient, in the case of offshore locations, varies between 0.01 in nearshore areas with onshore wind and 0.0001 in open sea without waves. For the study area, a conservative value of $z_{0}=0.001$ has been chosen. In this paper, the average annual offshore wave power has been calculated for $10 \mathrm{~m}$ and $100 \mathrm{~m}$. The electric power collected at the terminals is given by the following relation:

$$
P_{\text {wind }}=\frac{1}{2} C_{p}(w) \rho_{a} R_{b} w^{3}
$$

where $C_{p}(w)$ is the power coefficient and $R_{b}$ represents the area swept by the rotating blades. The maximum theoretical value of $C_{p}$, according to Betz's law, is 0.593 ; hence the maximum energy derived from a wind turbine is only the $59 \%$ of the available wind kinetic energy. Real values 
are lower, due to the losses in the turbine blades and PTO system. In this study, three practical utility-scale wind turbines have been analysed: a $100 \mathrm{~kW}$, a $1.0 \mathrm{MW}$ and a $2.3 \mathrm{MW}$ wind turbine generator (WTG). For each of the three class, the computation of estimated production curves are obtained averaging the performance curves provided by well-known wind turbine manufacturers (i.e., Northern Power System, Polaris America and Wind Energy Solutions for the $100 \mathrm{~kW}$ turbine; WinWind and Nordic Windpower for the 1.0 MW turbine; General Electric and Siemens for the 2.3 MW generator). The resulting power curves are reported in Appendix A. Moreover, the hub height $(z)$ is $80 \mathrm{~m}$ for the $2.3 \mathrm{MW}$ turbine, $55 \mathrm{~m}$ for $1 \mathrm{MW}$ generator and $25 \mathrm{~m}$ in $100 \mathrm{~kW}$ nacelle. Operatively, from 6-h pairs of wind component for $10 \mathrm{~m}$ height $\left(u_{10}, v_{10}\right)$, the wind speeds $w_{10}$ at the three hub heights have been computed and then, the Equation (10) has been applied to each swept areas to compute the electric power collected at the terminals of each model.

\section{Results}

\subsection{Wave Power Assessment}

In Tables 1 and 2 the geographical information of the grid points, such as the water depth and coordinates, and the main wave parameters, such as maximum, mean and standard deviation of the $H_{s}, T_{m}$ and $\theta_{m}$, have been calculated for each ECMWF grid point. The average significant wave height, $H_{s, \text { mean }}$, ranges between $1.3 \mathrm{~m}$ (E1) to $1.5 \mathrm{~m}$ (E5 and W5), while the average spectral mean period, $T_{m \text {,mean }}$, increases with the increasing of the mean wave height, varying between $7.81 \mathrm{~s}$ (E1) to $9.12 \mathrm{~s}$ (W5). It can be noted that the selected statistic wave parameters increase moving from North to South, but also a longitude influence can be noticed. The enhancing in wave characteristics is much more evident for extreme events. Indeed, the maximum significant wave height, $H_{s, \max }$, for western and eastern EMCWF points are respectively $3.59 \mathrm{~m}$ (W1) and 3.05 (E1). Also, the maximum mean period, $T_{m, \max }$, is smaller for eastern grid points (in average about $2.5 \mathrm{~s}$ ). The main reason for these reductions in wave parameters is the prevailing S-SW wave direction, which implies less wave energy per unit length of coastline relative to the east part of the archipelago. In this vein, the change from $195^{\circ}$ to $165^{\circ}$ in averaged mean direction, $\theta_{m, \text { mean }}$, moving from western to eastern reference points can also be explained.

The results of the yearly average wave power and wave energy, based on 10-year average at the 10 ECMWF grid points, are synthetized in Table 3. The annual average wave power was found to range between $8.46 \mathrm{~kW} / \mathrm{m}$ (E1) and $12.75 \mathrm{~kW} / \mathrm{m}$ (W5), while the annual average wave energy ranges between $99.23 \mathrm{MWh} / \mathrm{m}$ (W1) and $111.72 \mathrm{MWh} / \mathrm{m}$ (W5). Results of the analysis are graphically represented for each site with polar diagrams and scatter diagrams characterizing the energy source for different classes of significant wave height $\left(H_{m 0}\right)$ and energy period $\left(T_{e}\right)$, assembled in Figures 2 and 3.

Regarding the seasonal variability of the wave resource, small standard deviations of significant wave height $\sigma\left(H_{s}\right)$ and mean period $\sigma\left(T_{m}\right)$ indicate a low variability around a mean wave climate (Tables 1 and 2). Conversely, as confirmed by the average monthly power at each point in Tables 4 and 5 , a relatively high seasonal variability can be highlighted with mean values of the ratio between the minimum and mean average monthly wave power ranging from 0.53 for western to 0.60 for eastern points. In order to better visualize the wave energy resource, an offshore energy flux density contour line along the two vertical lines of the ECMWF points was computed and shown in Figure 4. For all grid points, waves with periods greater than $11 \mathrm{~s}$ cover a consistent amount of energy $(>20 \%)$, in accordance with the long fetch facing the archipelago. The Maldives, therefore, are extremely subjected to direct approach of swells from distant storms of the most perturbed region of the Indian Ocean (e.g., offshore of northern Madagascar). 
Table 1. Geographical information and main wave climate parameters (based on 10-year average) at points W1-W5.

\begin{tabular}{|c|c|c|c|c|c|c|c|c|c|c|c|}
\hline Point & Lat & Lon & $\begin{array}{c}\text { Depth } \\
(m)\end{array}$ & $\begin{array}{c}H_{s, \text { mean }} \\
(\mathrm{m})\end{array}$ & $\begin{array}{c}H_{s, \max } \\
(\mathrm{m})\end{array}$ & $\begin{array}{l}\sigma_{H s} \\
(m)\end{array}$ & $\begin{array}{c}T_{m, \text { mean }} \\
\text { (s) }\end{array}$ & $\begin{array}{c}T_{m, \max } \\
\text { (s) }\end{array}$ & $\begin{array}{c}\sigma_{T m} \\
(s)\end{array}$ & $\begin{array}{c}T_{e, \text { mean }} \\
(s)\end{array}$ & $\begin{array}{l}\Theta_{m} \\
\left(^{\circ}\right)\end{array}$ \\
\hline W1 & $4^{\circ} 30^{\prime} \mathrm{N}$ & $72^{\circ} 00^{\prime} \mathrm{E}$ & $>500$ & 1.41 & 3.59 & 0.18 & 8.92 & 15.06 & 1.90 & 8.91 & 202.54 \\
\hline W2 & $3^{\circ} 45^{\prime} \mathrm{N}$ & $72^{\circ} 00^{\prime} \mathrm{E}$ & $>500$ & 1.42 & 3.56 & 0.16 & 8.96 & 15.19 & 1.91 & 9.14 & 199.29 \\
\hline W3 & $3^{\circ} 00^{\prime} \mathrm{N}$ & $72^{\circ} 00^{\prime} \mathrm{E}$ & $>500$ & 1.45 & 3.45 & 0.14 & 9.01 & 15.19 & 1.87 & 9.48 & 194.87 \\
\hline W4 & $2^{\circ} 15^{\prime} \mathrm{N}$ & $72^{\circ} 00^{\prime} \mathrm{E}$ & $>500$ & 1.48 & 3.24 & 0.14 & 9.08 & 15.08 & 1.78 & 9.77 & 191.07 \\
\hline W5 & $1^{\circ} 30^{\prime} \mathrm{N}$ & $72^{\circ} 00^{\prime} \mathrm{E}$ & $>500$ & 1.51 & 3.07 & 0.13 & 9.12 & 14.96 & 1.68 & 10.00 & 187.39 \\
\hline \multicolumn{4}{|c|}{ Mean } & 1.45 & 3.38 & 0.15 & 9.02 & 15.09 & 1.83 & 10.28 & 195.03 \\
\hline
\end{tabular}

Table 2. Geographical information and main wave climate parameters (based on 10-year average) at points E1-E5.

\begin{tabular}{|c|c|c|c|c|c|c|c|c|c|c|c|}
\hline Point & Lat & Lon & $\begin{array}{c}\text { Depth } \\
(m)\end{array}$ & $\begin{array}{c}H_{s, \text { mean }} \\
(m)\end{array}$ & $\begin{array}{c}H_{s, \max } \\
(m)\end{array}$ & $\sigma_{H s}(m)$ & $\begin{array}{c}T_{m, m e a n} \\
(s)\end{array}$ & $\begin{array}{l}T_{m, \max } \\
\quad(s)\end{array}$ & $\begin{array}{c}\sigma_{T m} \\
(s)\end{array}$ & $\begin{array}{l}T_{e, \text { mean }} \\
(s)\end{array}$ & $\begin{array}{l}\Theta_{m} \\
\left(^{\circ}\right)\end{array}$ \\
\hline E1 & $4^{\circ} 30^{\prime} \mathrm{N}$ & $74^{\circ} 15^{\prime} \mathrm{E}$ & $>500$ & 1.33 & 3.05 & 0.11 & 7.81 & 11.95 & 1.11 & 8.91 & 165.36 \\
\hline E2 & $3^{\circ} 45^{\prime} \mathrm{N}$ & $74^{\circ} 15^{\prime} \mathrm{E}$ & $>500$ & 1.37 & 2.96 & 0.11 & 8.02 & 12.20 & 1.18 & 9.14 & 163.34 \\
\hline E3 & $3^{\circ} 00^{\prime} \mathrm{N}$ & $74^{\circ} 15^{\prime} \mathrm{E}$ & $>500$ & 1.42 & 2.87 & 0.11 & 8.32 & 12.72 & 1.29 & 9.48 & 163.15 \\
\hline E4 & $2^{\circ} 15^{\prime} \mathrm{N}$ & $74^{\circ} 15^{\prime} \mathrm{E}$ & $>500$ & 1.47 & 2.82 & 0.12 & 8.57 & 13.07 & 1.32 & 9.77 & 164.67 \\
\hline E5 & $1^{\circ} 30^{\prime} \mathrm{N}$ & $74^{\circ} 15^{\prime} \mathrm{E}$ & $>500$ & 1.53 & 2.80 & 0.12 & 8.77 & 13.35 & 1.33 & 10.00 & 166.48 \\
\hline \multicolumn{4}{|c|}{ Mean } & 1.42 & 2.90 & 0.11 & 8.30 & 12.66 & 1.25 & 9.46 & 164.60 \\
\hline
\end{tabular}

Table 3. Yearly average wave power and wave energy (based on 10-year average) at ECMWF grid points.

\begin{tabular}{cccccc}
\hline Point & $\begin{array}{c}\text { Average Power } \\
\mathbf{( k W / m )}\end{array}$ & $\begin{array}{c}\text { Yearly Energy } \\
\mathbf{( M W h / m )}\end{array}$ & Point & $\begin{array}{c}\text { Average Power } \\
(\mathbf{k W} / \mathbf{m})\end{array}$ & $\begin{array}{c}\text { Yearly Energy } \\
\mathbf{( M W h} / \mathbf{m})\end{array}$ \\
\hline W1 & 11.33 & 99.23 & E1 & 8.46 & 74.11 \\
W2 & 11.42 & 100.02 & E2 & 9.11 & 79.81 \\
W3 & 11.72 & 102.68 & E3 & 10.18 & 89.22 \\
W4 & 12.21 & 106.99 & $\mathbf{E 4}$ & 11.31 & 99.03 \\
W5 & 12.75 & 111.72 & E5 & 12.45 & 109.07 \\
\hline Mean & 11.89 & 104.13 & Mean & 10.30 & 90.25 \\
\hline
\end{tabular}
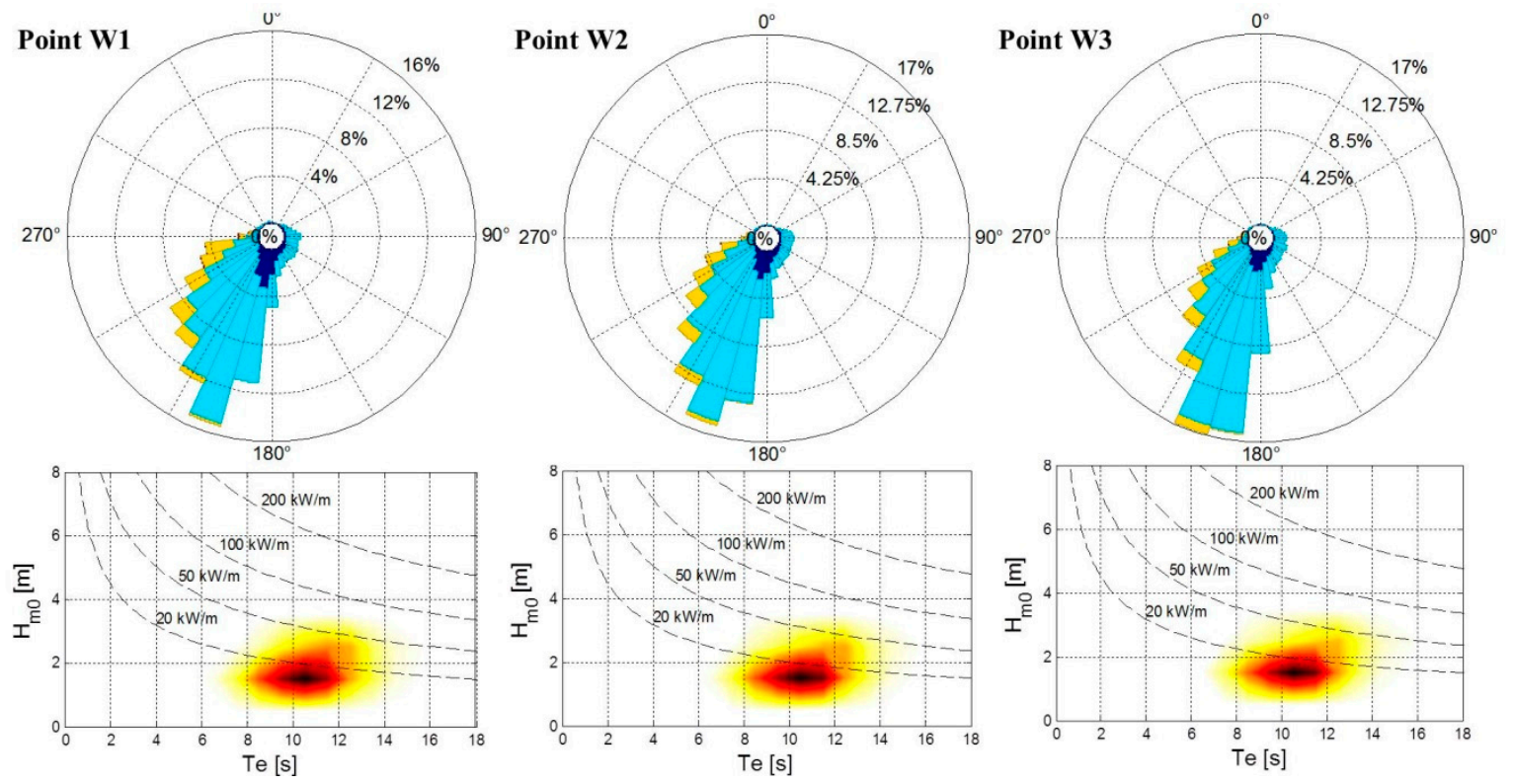

Figure 2. Cont. 


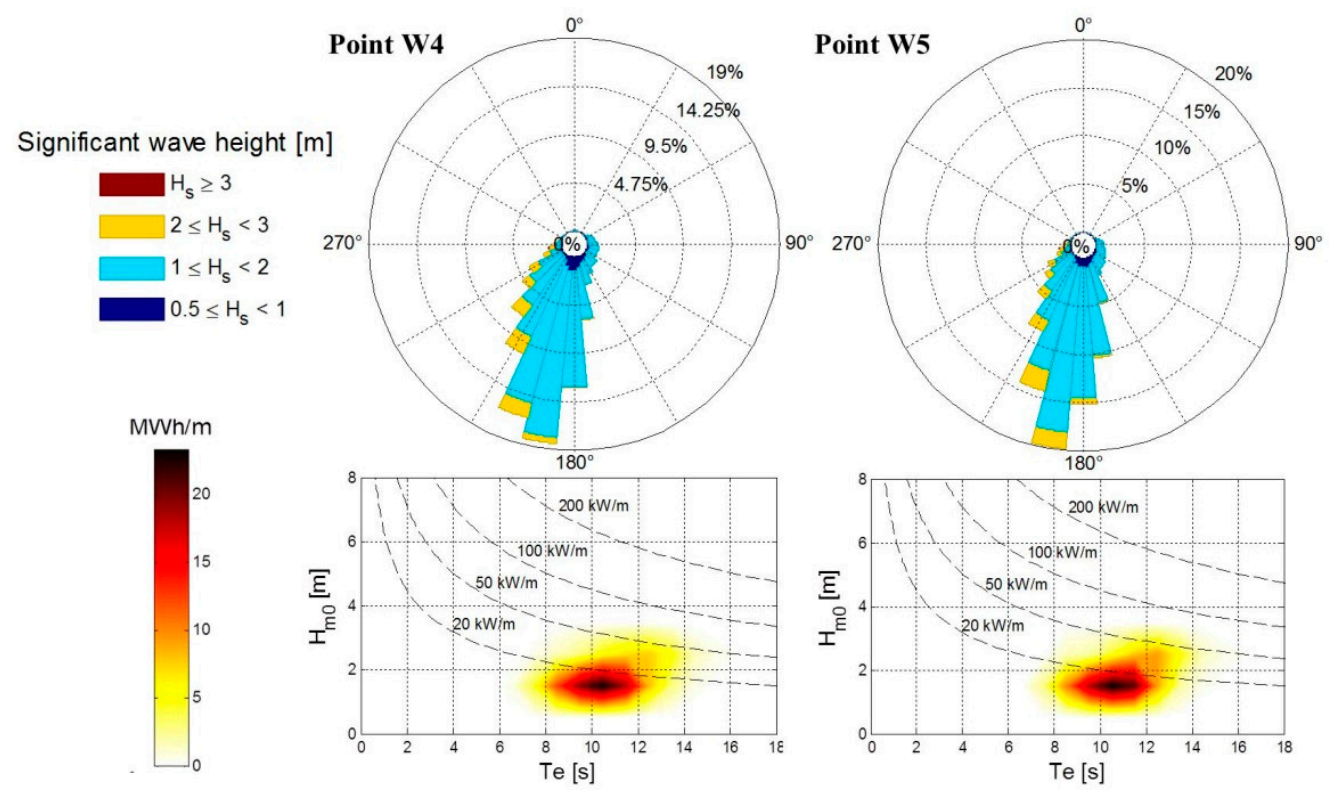

Figure 2. Characterization of the yearly average wave energy points E1-E5 in terms of significant wave height $\left(H_{m 0}\right)$ and energy period $\left(T_{e}\right)$. The colors scale represents annual energy per meter of wave front (in $\mathrm{MWh} / \mathrm{m}$ ) and the isolines refer to wave power.
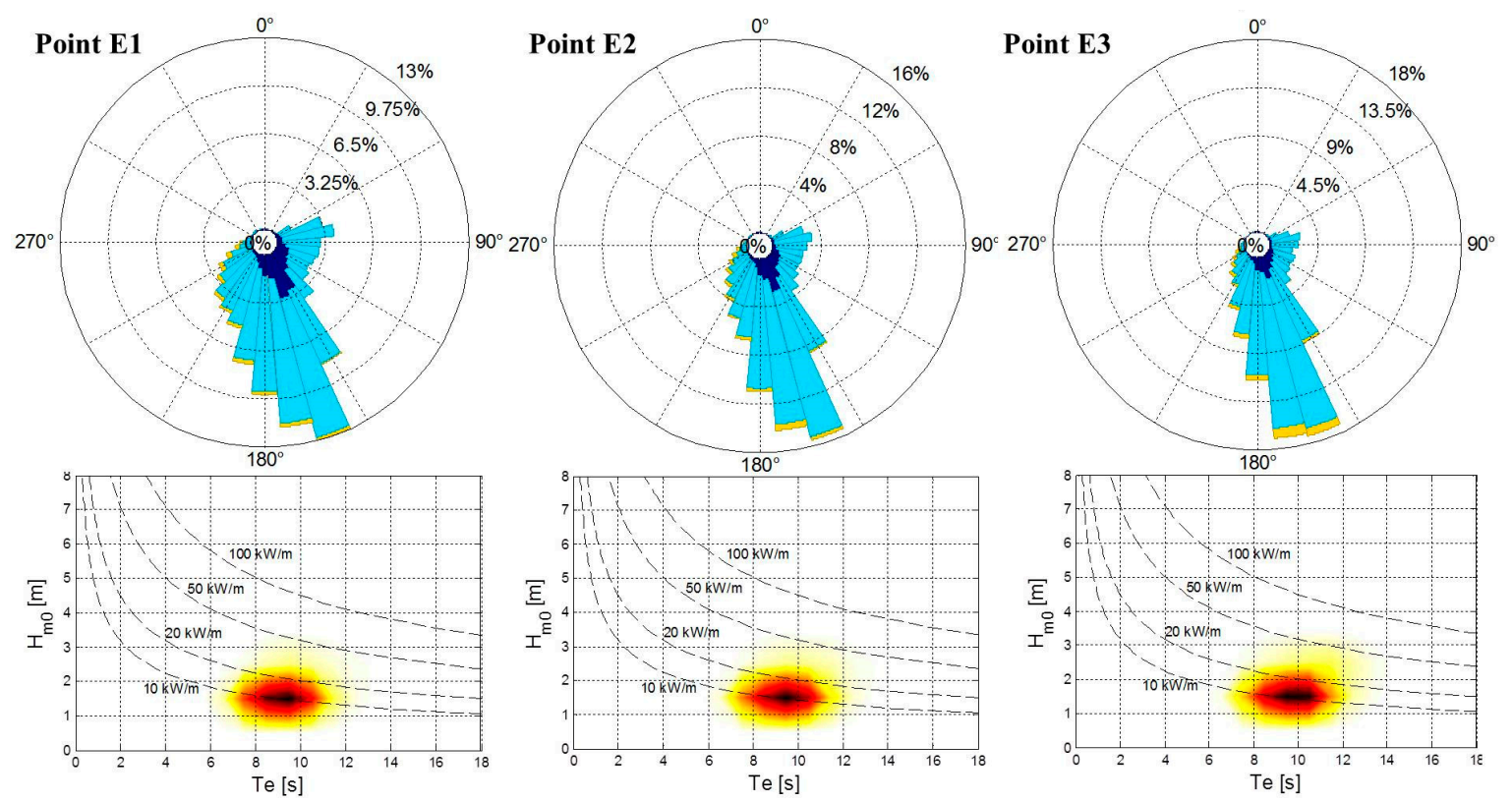

Figure 3. Cont. 

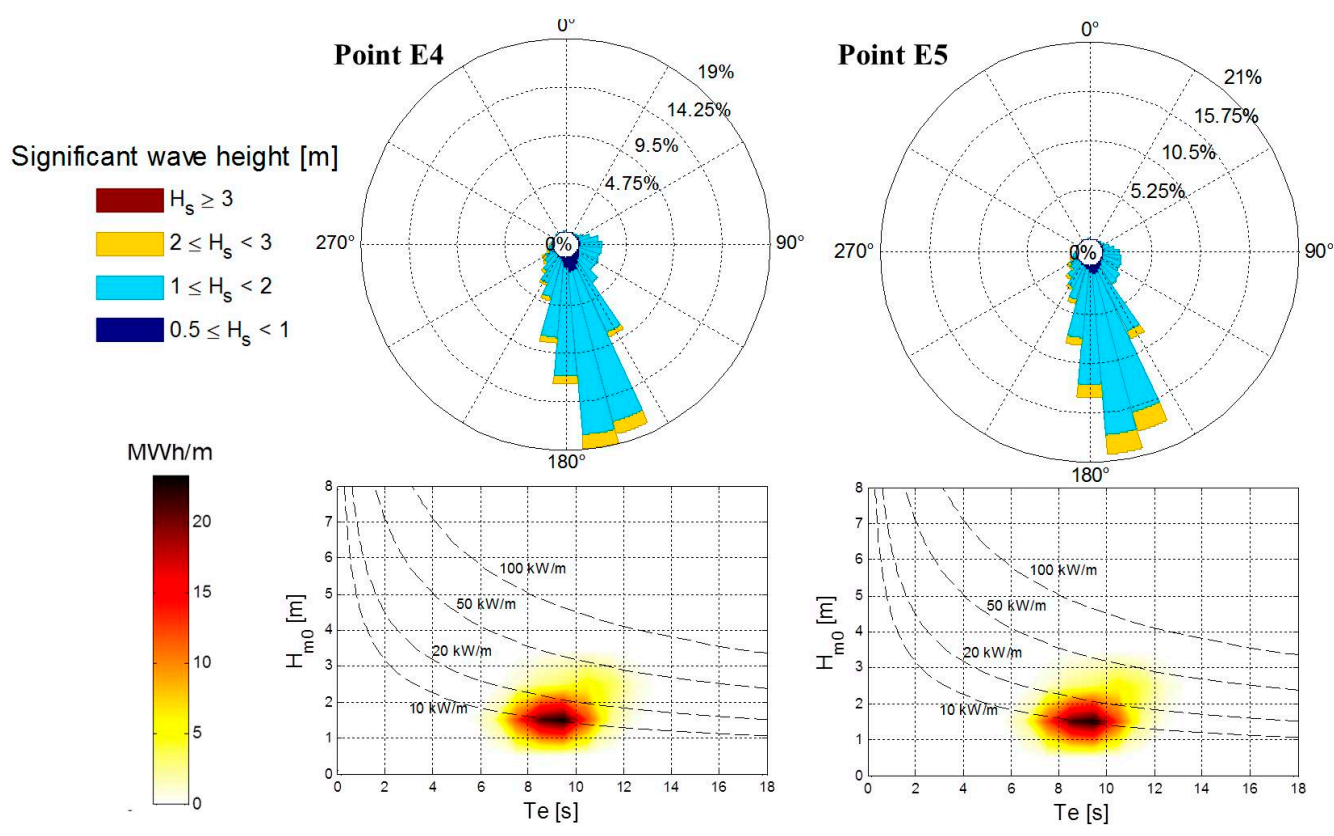

Figure 3. Characterization of the yearly average wave energy points E1-E5 in terms of significant wave height $\left(H_{m 0}\right)$ and energy period $\left(T_{e}\right)$. The colors scale represents annual energy per meter of wave front (in $\mathrm{MWh} / \mathrm{m}$ ) and the isolines refer to wave power.

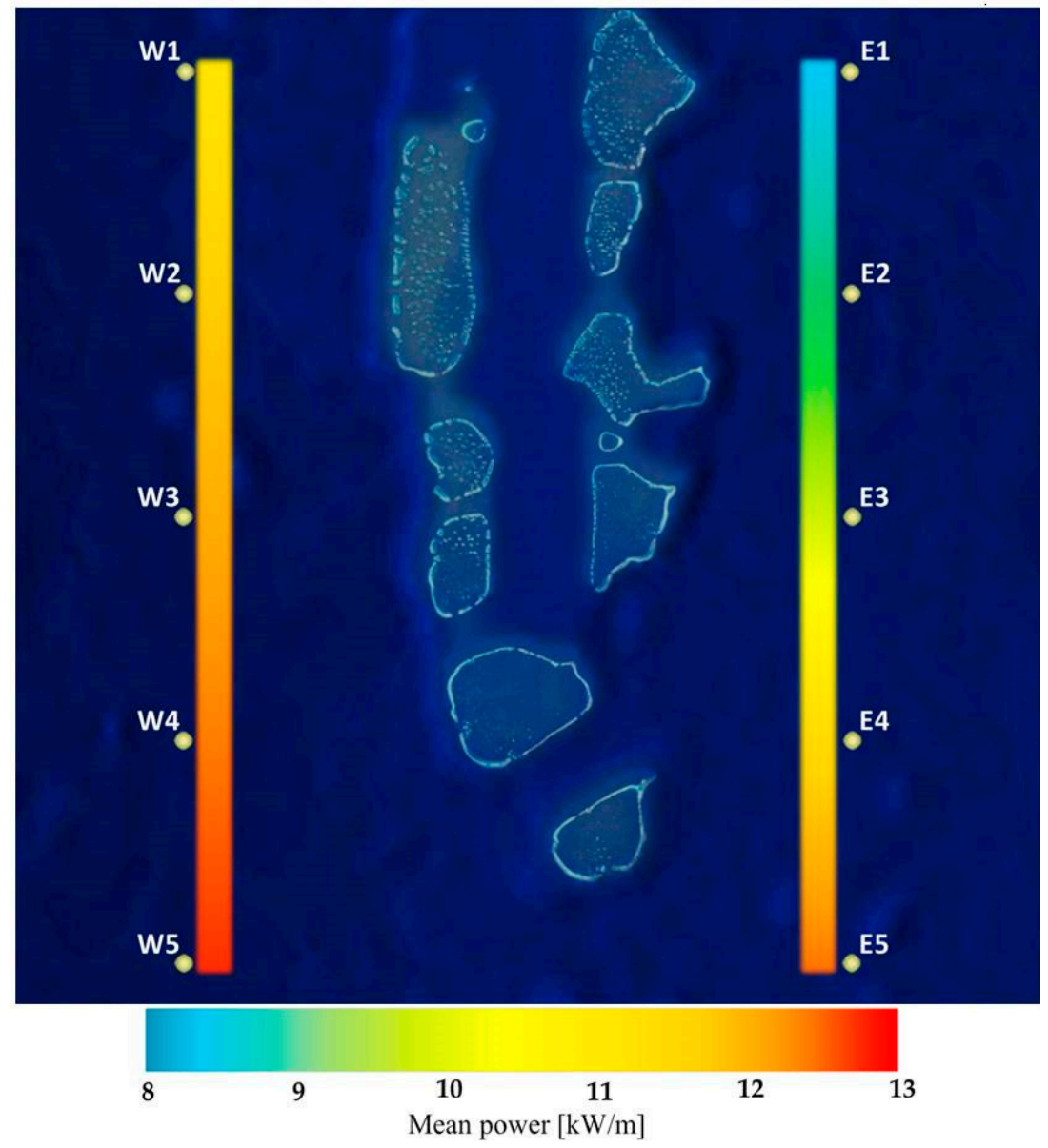

Figure 4. Mean wave power flux per unit crest along the 10 ECMWF offshore points. 
Table 4. Monthly wave power (based on 10-year average) at ECMWF grid points W1-W5.

\begin{tabular}{|c|c|c|c|c|c|c|c|c|c|c|c|c|c|}
\hline \multirow{2}{*}{ Point } & \multicolumn{12}{|c|}{ Average Monthly Power (kW/m) } & \multirow[b]{2}{*}{ Min/Mean } \\
\hline & January & February & March & April & May & June & July & August & September & October & November & December & \\
\hline W1 & 6.1 & 5.6 & 5.7 & 7.4 & 11.7 & 21.3 & 21.6 & 16.4 & 14.6 & 11.3 & 7.4 & 6.4 & 0.49 \\
\hline W2 & 6.4 & 5.8 & 6.0 & 7.8 & 12.1 & 20.5 & 20.8 & 16.3 & 14.8 & 11.8 & 7.7 & 6.7 & 0.51 \\
\hline W3 & 7.0 & 6.2 & 6.4 & 8.4 & 12.6 & 20.0 & 20.5 & 16.6 & 15.2 & 12.4 & 8.3 & 7.1 & 0.53 \\
\hline W4 & 7.0 & 6.6 & 6.8 & 9.0 & 13.2 & 19.9 & 20.8 & 17.3 & 16.0 & 13.0 & 8.9 & 7.5 & 0.54 \\
\hline W5 & 7.4 & 7.0 & 7.3 & 9.7 & 13.8 & 20.0 & 21.3 & 18.1 & 16.9 & 13.7 & 9.4 & 8.0 & 0.55 \\
\hline Mean & 6.8 & 6.2 & 6.4 & 8.5 & 12.7 & 20.3 & 21.0 & 16.9 & 15.5 & 12.4 & 8.3 & 7.2 & 0.53 \\
\hline
\end{tabular}

Table 5. Monthly wave power (based on 10-year average) at ECMWF grid points E1-E5.

\begin{tabular}{|c|c|c|c|c|c|c|c|c|c|c|c|c|c|}
\hline \multirow{2}{*}{ Point } & \multicolumn{12}{|c|}{ Average Monthly Power (kW/m) } & \multirow[b]{2}{*}{ Min/Mean } \\
\hline & January & February & March & April & May & June & July & August & September & October & November & December & \\
\hline E1 & 6.0 & 5.3 & 5.0 & 5.7 & 8.5 & 12.4 & 14.5 & 12.7 & 11.6 & 8.2 & 5.6 & 5.9 & 0.59 \\
\hline E2 & 6.3 & 5.6 & 5.5 & 6.4 & 9.4 & 13.0 & 15.3 & 13.7 & 12.5 & 9.0 & 6.2 & 6.2 & 0.60 \\
\hline E3 & 6.7 & 6.2 & 6.2 & 7.4 & 10.7 & 14.4 & 16.9 & 15.4 & 14.0 & 10.3 & 7.0 & 6.6 & 0.61 \\
\hline E4 & 7.2 & 6.7 & 6.9 & 8.4 & 12.0 & 16.0 & 18.7 & 17.0 & 15.6 & 11.6 & 8.0 & 7.2 & 0.59 \\
\hline E5 & 7.7 & 7.3 & 7.7 & 9.5 & 13.3 & 17.7 & 20.5 & 18.7 & 17.1 & 12.9 & 8.9 & 7.8 & 0.58 \\
\hline Mean & 6.8 & 6.2 & 6.2 & 7.5 & 10.8 & 14.7 & 17.2 & 15.5 & 14.1 & 10.4 & 7.1 & 6.7 & 0.60 \\
\hline
\end{tabular}




\subsection{Wind Power Assessment}

The results show that windiness increases in the northeastern area. For point E1, in fact, the average annual wind power density, $P_{\text {wind,mean }}$, raises from $104 \mathrm{~W} / \mathrm{m}^{2}$ to $204 \mathrm{~W} / \mathrm{m}^{2}$ at a height of $10 \mathrm{~m}$ and $100 \mathrm{~m}$ respectively, as shown in Tables 6 and 7, in which the wind power at the reference heights of 25,55 and $80 \mathrm{~m}$ are also compared.

Table 6. The average annual wind power density at points W1-W5.

\begin{tabular}{cccccc}
\hline \multirow{2}{*}{ Point } & \multicolumn{5}{c}{ P $_{\text {wind_MEAN }}\left(\mathbf{W} / \mathbf{m}^{\mathbf{2}}\right)$} \\
\cline { 2 - 6 } & $\mathbf{1 0 ~} \mathbf{~}$ & $\mathbf{2 5} \mathbf{~ m}$ & $\mathbf{5 5} \mathbf{~}$ & $\mathbf{8 0 ~} \mathbf{~}$ & $\mathbf{1 0 0} \mathbf{~}$ \\
\hline W1 & 88.0 & 117.0 & 146.5 & 162.1 & 171.9 \\
W2 & 84.6 & 112.4 & 140.7 & 155.5 & 165.2 \\
W3 & 79.9 & 106.2 & 133.0 & 147.2 & 156.1 \\
W4 & 75.5 & 100.4 & 125.7 & 139.2 & 147.6 \\
W5 & 71.8 & 95.4 & 119.4 & 132.2 & 140.1 \\
\hline Mean & 80.0 & 106.3 & 133.1 & 147.3 & 156.2 \\
\hline
\end{tabular}

Table 7. The average annual wind power density at points E1-E5.

\begin{tabular}{cccccc}
\hline \multirow{2}{*}{ Point } & \multicolumn{5}{c}{ P $_{\text {wind_MEAN }}\left(\mathbf{W} / \mathbf{m}^{\mathbf{2}}\right)$} \\
\cline { 2 - 6 } & $\mathbf{1 0 ~} \mathbf{~}$ & $\mathbf{2 5} \mathbf{~ m}$ & $\mathbf{5 5} \mathbf{~}$ & $\mathbf{8 0 ~} \mathbf{~}$ & $\mathbf{1 0 0} \mathbf{~}$ \\
\hline E1 & 104.4 & 138.6 & 173.7 & 192.3 & 203.9 \\
E2 & 97.0 & 129.0 & 161.5 & 178.7 & 189.4 \\
E3 & 89.2 & 118.6 & 148.5 & 164.2 & 174.3 \\
E4 & 81.7 & 108.5 & 136.0 & 150.5 & 159.6 \\
E5 & 75.6 & 100.6 & 126.0 & 139.4 & 147.8 \\
\hline Mean & 89.6 & 119.1 & 149.1 & 165.0 & 175.0 \\
\hline
\end{tabular}

For all of these reference heights, wind parameters, such as minimum, maximum, mean and standard deviation of wind speed $\left(W_{\text {min }}, W_{\text {max }}, W_{\text {mean }}\right.$ and $\sigma(W)$ respectively), have been calculated and reported in Tables 7 and 8.

In order to quantify the energy fluctuations and the inactivity periods of the wind plants, a working time parameter, $T_{w}$, is reported, calculated as the fraction of a year in which a wind plant could yield energy, i.e., the wind speed is within the cut-in $\left(w_{\text {in }}\right)$ and cut-off $\left(w_{\text {off }}\right)$ speed. In the present study, these two working limits have been assumed to be equal to $3 \mathrm{~m} / \mathrm{s}$ and $18 \mathrm{~m} / \mathrm{s}$ respectively. In this vein, to provide information about the temporal distribution of the collected energy, the exploitable power fraction, $X_{w}$, is also shown (Tables 8 and 9). The latter parameter represents the fraction of the total wind power which may be exploited by a wind generator during operation conditions (i.e., when $\left.\mathrm{w}_{\text {in }}<\mathrm{w}<\mathrm{w}_{\text {off }}\right)$. All the ECMWF points are characterized by a windiness that would allow a wind turbine to supply energy quite continuously over time. Referring to the standard height of $10 \mathrm{~m}$, with the above-mentioned values of the cut-in and cut-off speed, the working time varies from $73.7 \%$ at W5 to $76.8 \%$ at W1. It is highlighted the very high value of exploitable power fraction $\left(X_{\mathrm{w}}>97 \%\right)$ for all sites at every reference height. It is worth noting also that maximum hindcast wind speed is $16.46 \mathrm{~m} / \mathrm{s}$ (W5 at $100 \mathrm{~m}$ ). Albeit this is not the true maximum wind speed measurable in the area, since smaller sampling frequency for the hindcast data involves peak attenuation, it demonstrates as the area experiences great windiness regularity. 
Table 8. Main wind climate parameters (based on 10-year average) at points W1-W5.

\begin{tabular}{|c|c|c|c|c|c|c|c|}
\hline \multirow{2}{*}{ Reference Height } & \multirow{2}{*}{ Point } & \multirow{2}{*}{$\frac{W_{\min }}{(\mathrm{m} / \mathrm{s})}$} & \multirow{2}{*}{$\frac{W_{\max }}{(\mathrm{m} / \mathrm{s})}$} & \multirow{2}{*}{$\frac{W_{\text {mean }}}{(\mathrm{m} / \mathrm{s})}$} & \multirow{2}{*}{$\frac{\sigma_{W}}{(\mathrm{~m} / \mathrm{s})}$} & \multirow{2}{*}{$\mathrm{T}_{\mathrm{w}}(\%)$} & \multirow{2}{*}{$X_{w}(\%)$} \\
\hline & & & & & & & \\
\hline \multirow{5}{*}{$10 \mathrm{~m}$} & W1 & 0.06 & 13.06 & 4.55 & 1.99 & 76.79 & 98.46 \\
\hline & W2 & 0.03 & 12.95 & 4.50 & 1.95 & 76.46 & 98.22 \\
\hline & W3 & 0.03 & 12.58 & 4.44 & 1.92 & 75.53 & 97.89 \\
\hline & W4 & 0.05 & 12.73 & 4.37 & 1.91 & 74.75 & 97.64 \\
\hline & W5 & 0.03 & 13.17 & 4.31 & 1.89 & 73.72 & 97.32 \\
\hline \multirow{5}{*}{$25 \mathrm{~m}$} & W1 & 0.07 & 14.36 & 5.01 & 2.19 & 81.04 & 99.06 \\
\hline & W2 & 0.03 & 14.24 & 4.95 & 2.15 & 80.64 & 98.93 \\
\hline & W3 & 0.04 & 13.83 & 4.88 & 2.11 & 80.06 & 98.67 \\
\hline & W4 & 0.06 & 14.00 & 4.81 & 2.10 & 79.05 & 98.52 \\
\hline & W5 & 0.03 & 14.48 & 4.74 & 2.08 & 78.09 & 98.27 \\
\hline \multirow{5}{*}{$55 \mathrm{~m}$} & W1 & 0.07 & 15.48 & 5.39 & 2.36 & 83.76 & 99.39 \\
\hline & W2 & 0.03 & 15.35 & 5.34 & 2.32 & 83.35 & 99.24 \\
\hline & W3 & 0.04 & 14.91 & 5.26 & 2.28 & 83.08 & 99.14 \\
\hline & W4 & 0.06 & 15.09 & 5.18 & 2.26 & 81.98 & 98.99 \\
\hline & W5 & 0.03 & 15.61 & 5.11 & 2.24 & 81.41 & 98.83 \\
\hline \multirow{5}{*}{$80 \mathrm{~m}$} & W1 & 0.08 & 16.01 & 5.58 & 2.44 & 84.84 & 99.48 \\
\hline & W2 & 0.03 & 15.88 & 5.52 & 2.39 & 84.36 & 99.35 \\
\hline & W3 & 0.04 & 15.42 & 5.44 & 2.36 & 84.08 & 99.26 \\
\hline & W4 & 0.06 & 15.61 & 5.36 & 2.34 & 83.19 & 99.13 \\
\hline & W5 & 0.04 & 16.14 & 5.28 & 2.32 & 82.68 & 99.04 \\
\hline \multirow{5}{*}{$100 \mathrm{~m}$} & W1 & 0.08 & 16.33 & 5.69 & 2.49 & 85.32 & 99.53 \\
\hline & W2 & 0.03 & 16.19 & 5.63 & 2.44 & 84.97 & 99.41 \\
\hline & W3 & 0.04 & 15.72 & 5.55 & 2.40 & 84.62 & 99.34 \\
\hline & W4 & 0.07 & 15.92 & 5.47 & 2.38 & 83.92 & 99.21 \\
\hline & W5 & 0.04 & 16.46 & 5.39 & 2.37 & 83.52 & 99.13 \\
\hline
\end{tabular}

Table 9. Main wind climate parameters (based on 10-year average) at points E1-E5.

\begin{tabular}{|c|c|c|c|c|c|c|c|}
\hline \multirow{2}{*}{ Reference Height } & \multirow{2}{*}{ Point } & \multirow{2}{*}{$\begin{array}{l}W_{\min } \\
(\mathrm{m} / \mathrm{s})\end{array}$} & \multirow{2}{*}{$\begin{array}{l}W_{\max } \\
(\mathrm{m} / \mathrm{s})\end{array}$} & \multirow{2}{*}{$\begin{array}{c}W_{\text {mean }} \\
(\mathrm{m} / \mathrm{s})\end{array}$} & \multirow{2}{*}{$\frac{\sigma_{W}}{(\mathrm{~m} / \mathrm{s})}$} & \multirow{2}{*}{$\mathrm{T}_{\mathrm{w}}(\%)$} & \multirow{2}{*}{$X_{w}(\%)$} \\
\hline & & & & & & & \\
\hline \multirow{5}{*}{$10 \mathrm{~m}$} & E1 & 0.04 & 12.96 & 4.97 & 2.04 & 81.32 & 98.81 \\
\hline & E2 & 0.07 & 13.37 & 4.86 & 1.99 & 80.97 & 98.65 \\
\hline & E3 & 0.06 & 12.80 & 4.75 & 1.96 & 79.83 & 98.37 \\
\hline & E4 & 0.05 & 12.57 & 4.62 & 1.93 & 78.38 & 98.09 \\
\hline & E5 & 0.05 & 12.21 & 4.50 & 1.91 & 77.20 & 97.70 \\
\hline \multirow{5}{*}{$25 \mathrm{~m}$} & E1 & 0.04 & 14.25 & 5.46 & 2.24 & 84.89 & 99.32 \\
\hline & E2 & 0.07 & 14.70 & 5.35 & 2.19 & 84.39 & 99.20 \\
\hline & E3 & 0.07 & 14.07 & 5.22 & 2.15 & 83.54 & 99.00 \\
\hline & E4 & 0.05 & 13.82 & 5.08 & 2.12 & 82.47 & 98.84 \\
\hline & E5 & 0.05 & 13.43 & 4.95 & 2.10 & 81.43 & 98.57 \\
\hline \multirow{5}{*}{$55 \mathrm{~m}$} & E1 & 0.05 & 15.36 & 5.89 & 2.41 & 87.03 & 99.56 \\
\hline & E2 & 0.08 & 15.85 & 5.76 & 2.36 & 86.66 & 99.46 \\
\hline & E3 & 0.07 & 15.17 & 5.63 & 2.32 & 85.97 & 99.34 \\
\hline & E4 & 0.06 & 14.90 & 5.48 & 2.29 & 85.29 & 99.21 \\
\hline & E5 & 0.06 & 14.47 & 5.34 & 2.27 & 84.23 & 99.07 \\
\hline \multirow{5}{*}{$80 \mathrm{~m}$} & E1 & 0.05 & 15.89 & 6.09 & 2.50 & 87.88 & 99.60 \\
\hline & E2 & 0.08 & 16.39 & 5.96 & 2.44 & 87.69 & 99.57 \\
\hline & E3 & 0.08 & 15.69 & 5.82 & 2.40 & 86.95 & 99.45 \\
\hline & E4 & 0.06 & 15.41 & 5.66 & 2.37 & 86.29 & 99.33 \\
\hline & E5 & 0.06 & 14.97 & 5.52 & 2.34 & 85.46 & 99.23 \\
\hline \multirow{5}{*}{$100 \mathrm{~m}$} & E1 & 0.05 & 16.21 & 6.21 & 2.55 & 88.44 & 99.64 \\
\hline & E2 & 0.08 & 16.72 & 6.08 & 2.49 & 88.24 & 99.62 \\
\hline & E3 & 0.08 & 16.00 & 5.94 & 2.45 & 87.54 & 99.51 \\
\hline & E4 & 0.06 & 15.72 & 5.78 & 2.41 & 86.71 & 99.38 \\
\hline & E5 & 0.06 & 15.27 & 5.63 & 2.39 & 86.01 & 99.32 \\
\hline
\end{tabular}


This result is confirmed in Tables 10 and 11, where the monthly mean speeds at $10 \mathrm{~m}, U_{10, \text { mean }}$, have been summarized. For all the selected points, the ratio between the minimum and mean average monthly wind speed $U_{10 \text {,mean }}$ shows very high values, hence the area is characterized by very low seasonal wind speed variability. These outcomes seem in contrasts with some preliminary information described in [1], in which the Authors pointed out for the selected area long periods of the year with almost no useable wind.

Figures 5 and 6 report the wind rose with both the annual frequency distribution of the wind direction and the annual mean wind speed. It is clearly shown as the wind mostly blows from the West, with a secondary sector from E-NE. In order to better visualize the wind climate, an offshore energy flux density plot was computed and shown in Figure 7.
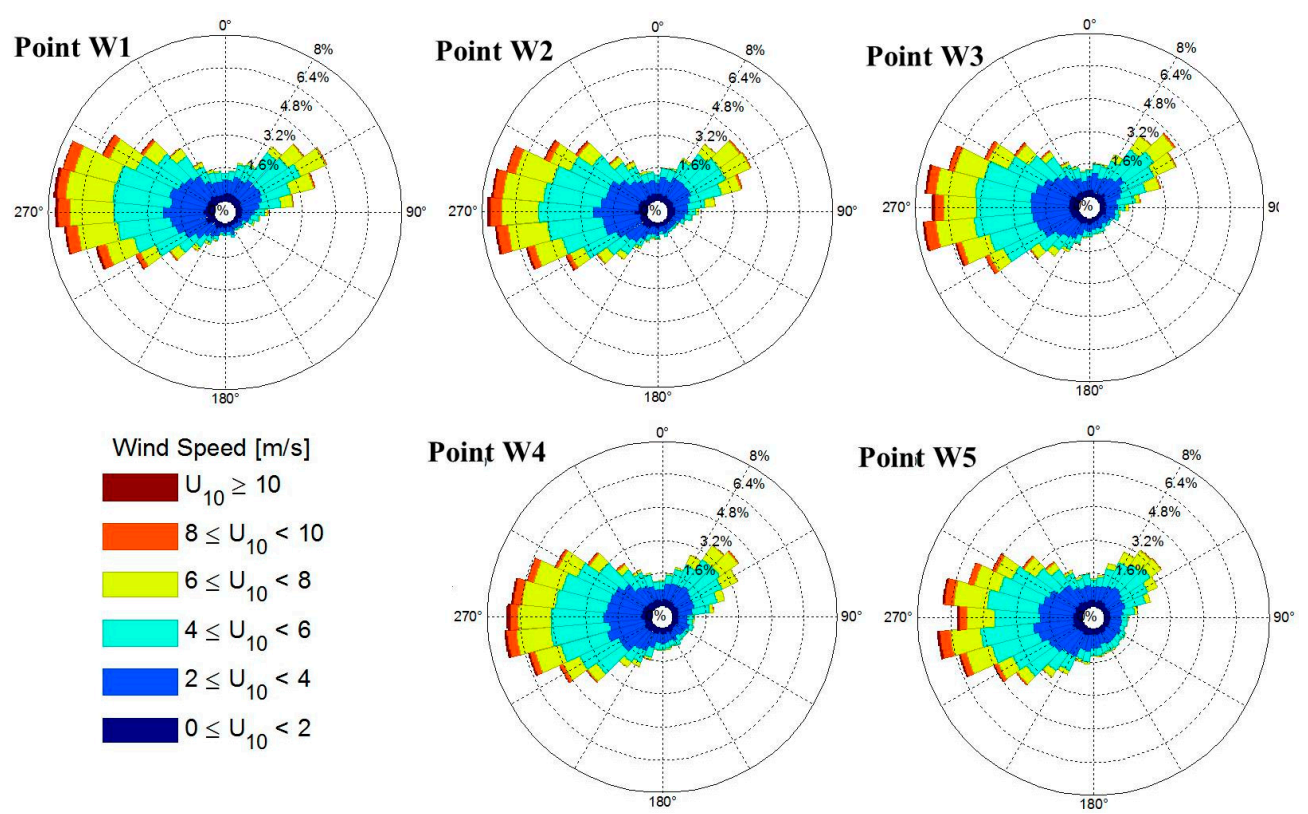

Figure 5. Wind rose at points W1-W5 with directional bins of $10^{\circ}$.

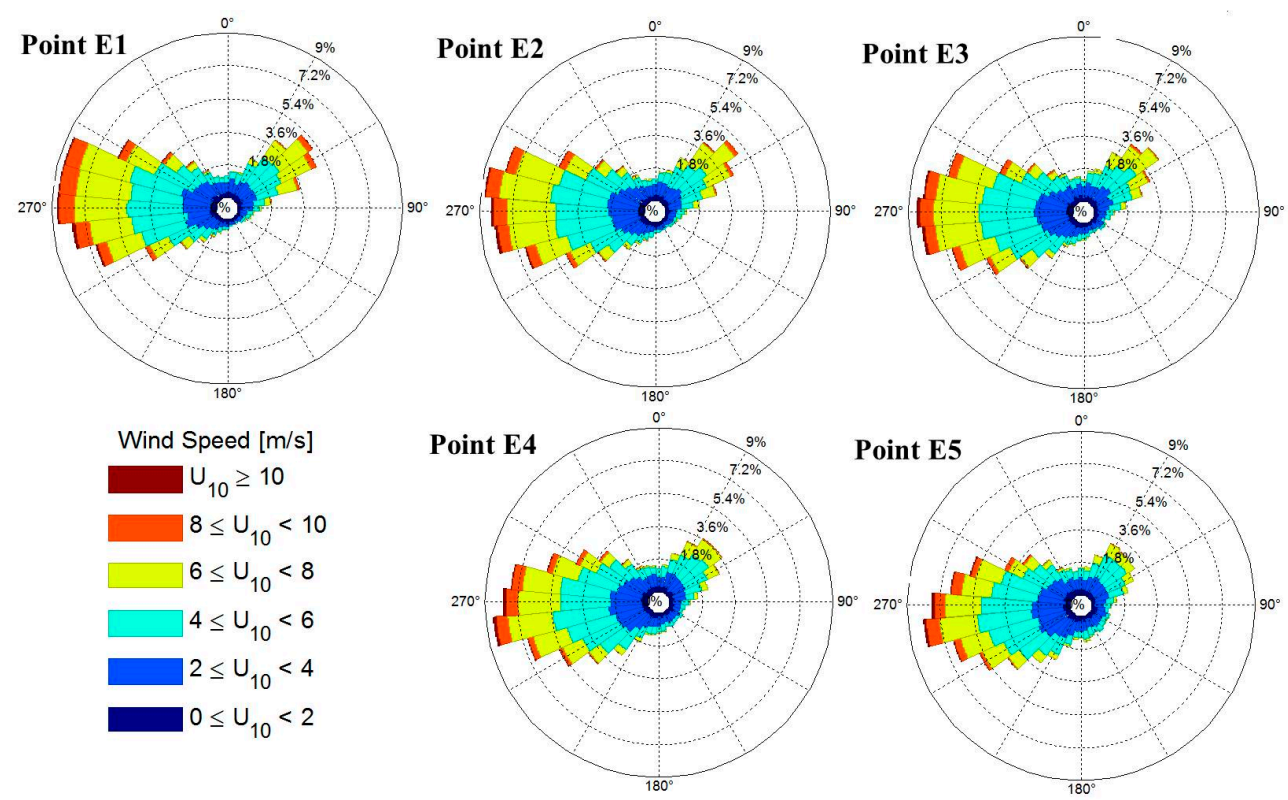

Figure 6. Wind rose at points E1-E5 with directional bins of $10^{\circ}$. 
Table 10. Monthly mean speeds $U_{10, \text { mean }}$ at points W1-W5.

\begin{tabular}{|c|c|c|c|c|c|c|c|c|c|c|c|c|c|}
\hline \multirow{2}{*}{ Point } & \multicolumn{11}{|c|}{$U_{10, \text { mean }}(\mathrm{m} / \mathrm{s})$} & \multirow[b]{2}{*}{ December } & \multirow[b]{2}{*}{ Min/Mean } \\
\hline & January & February & March & April & May & June & July & August & September & October & November & & \\
\hline W1 & 5.0 & 4.5 & 3.6 & 3.4 & 5.1 & 5.5 & 5.2 & 4.8 & 5.1 & 4.6 & 3.8 & 4.0 & 0.74 \\
\hline W2 & 5.1 & 4.6 & 3.6 & 3.5 & 5.1 & 5.2 & 4.8 & 4.5 & 4.9 & 4.7 & 4.0 & 4.1 & 0.78 \\
\hline W3 & 4.2 & 4.7 & 3.6 & 3.6 & 5.1 & 4.8 & 4.4 & 4.2 & 4.8 & 4.8 & 4.1 & 4.1 & 0.82 \\
\hline W4 & 4.9 & 4.6 & 3.6 & 3.8 & 5.0 & 4.5 & 4.1 & 3.9 & 4.6 & 4.9 & 4.4 & 4.2 & 0.82 \\
\hline W5 & 4.8 & 4.5 & 3.6 & 3.9 & 4.9 & 4.2 & 3.9 & 3.7 & 4.4 & 4.9 & 4.6 & 4.3 & 0.83 \\
\hline Mean & 6.8 & 6.2 & 6.2 & 7.5 & 10.8 & 14.7 & 17.2 & 15.5 & 14.1 & 10.4 & 7.1 & 6.7 & 0.80 \\
\hline
\end{tabular}

Table 11. Monthly mean speeds $U_{10, \text { mean }}$ at points E1-E5.

\begin{tabular}{|c|c|c|c|c|c|c|c|c|c|c|c|c|c|}
\hline Points & January & February & March & April & May & June & July & August & September & October & November & December & Min/Mean \\
\hline E1 & 5.5 & 4.8 & 3.7 & 3.8 & 5.8 & 6.0 & 5.7 & 5.5 & 5.5 & 4.8 & 4.1 & 4.5 & 0.74 \\
\hline E2 & 5.5 & 4.9 & 3.7 & 3.9 & 5.8 & 5.6 & 5.2 & 5.1 & 5.2 & 4.9 & 4.2 & 4.5 & 0.75 \\
\hline E3 & 4.4 & 4.8 & 3.6 & 4.0 & 5.7 & 5.2 & 4.8 & 4.8 & 5.1 & 5.0 & 4.3 & 4.4 & 0.78 \\
\hline E4 & 5.1 & 4.6 & 3.6 & 4.1 & 5.5 & 4.9 & 4.5 & 4.6 & 4.9 & 5.0 & 4.5 & 4.3 & 0.78 \\
\hline E5 & 4.8 & 4.5 & 3.6 & 4.2 & 5.3 & 4.6 & 4.2 & 4.3 & 4.7 & 4.9 & 4.6 & 4.4 & 0.79 \\
\hline Mean & 6.8 & 6.2 & 6.2 & 7.5 & 10.8 & 14.7 & 17.2 & 15.5 & 14.1 & 10.4 & 7.1 & 6.7 & 0.77 \\
\hline
\end{tabular}




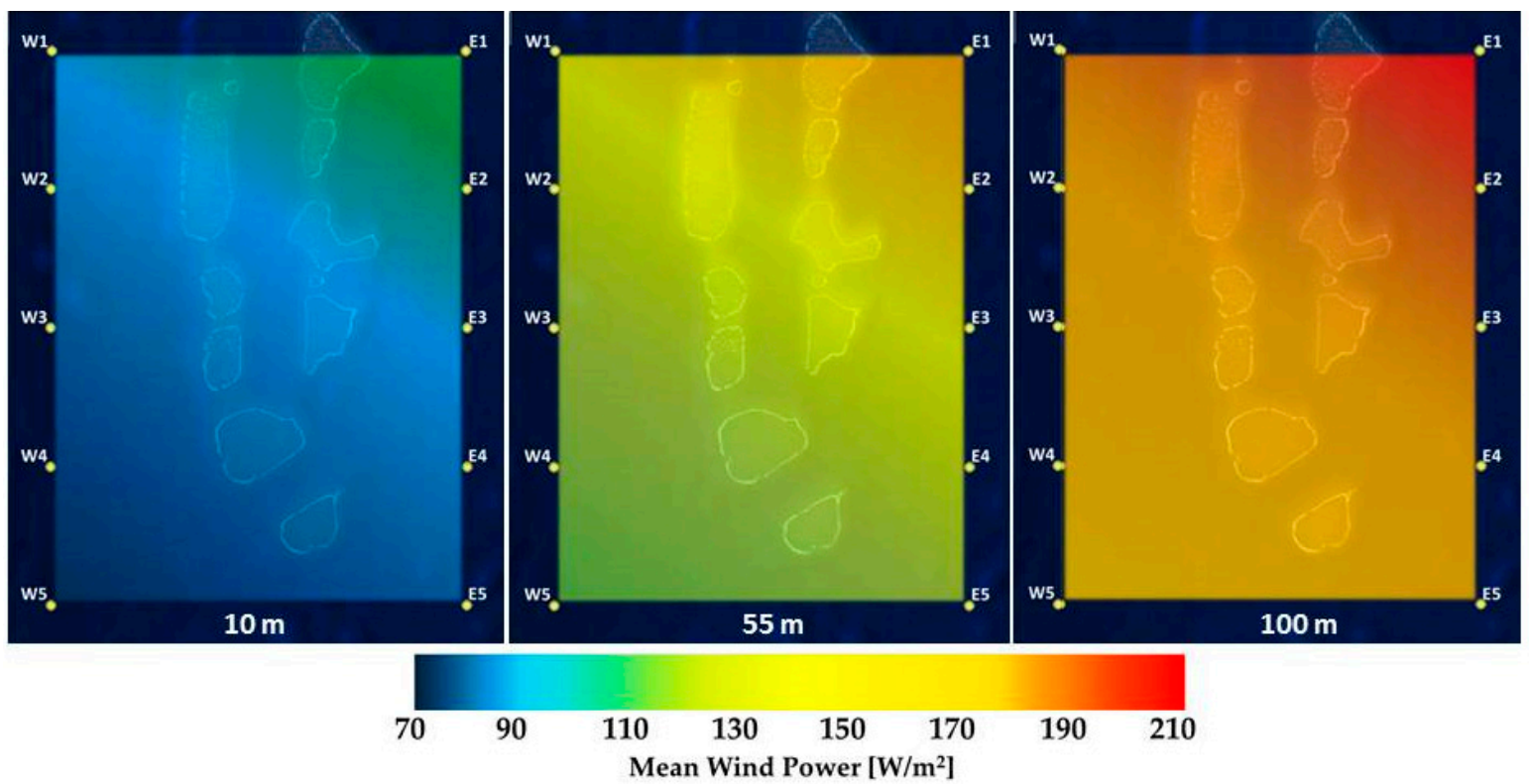

Figure 7. Mean wind power plot along the studied area.

\section{Additional Considerations and Discussion}

As showed in previous chapter, the area is characterized by a significant seasonality of wave regimes and a poor monthly variability of wind conditions. An heuristic explanation could be provided considering that the Maldives wave sea state is comprised of multiple wave fields, i.e., sea waves generated by local winds plus very long-period swells approaching from several distant sources and different directions. In support of this argument, it is possible to note as the highest values of wave power are measured from May to October, which correspond to the period when the southwest monsoon is in full swing [72]. On the basis of the above considerations, it is reasonable to also explain the poor relationship between the main directions of waves and local winds. As demonstrate by [73], the time scale of the directional response appears to be equal to the time scale of wave energy growth only for young sea states, hence in absence of swell. Therefore, the energy patterns suggest that wind and waves are generally uncorrelated, especially during the period of southwest monsoon. These conditions are very interesting in the perspective of reducing the overall variability of the produced power. Indeed, the correlation of ocean waves to wind is a very important aspect for both forecasting models of energy production and for design of mooring/substructure/structure of wind/wave energy devices.

In order to boost blue growth and make wind and wave energies environmentally and socio-economically sustainable, a role of preference could be addressed bringing together the energy demand with other local issues. Indeed, the need for fresh water is at the top of the Government agenda for critical problems. Malé, the capital of the Maldives, declared a state of emergency in 2014 due to the lack of potable water, when the majority of the inhabitants were left without access to potable water. Today, rainwater is more widely used than groundwater for drinking purpose and about $88 \%$ of the population in Malè intercepts rainwater via tanks [1]. Furthermore, it is expected that population growth and the increase of the number of tourists will result in ever-increasing demands for clean water. Generally, the potable water demand in coastal areas is met by means of large-scale desalination systems. However, also the modern systems require very high energy consumption, which still represents a crucial drawback for this technology. Furthermore, numerous low-density population islands lack not only fresh water availability, but also the electrical grid connection or any other energy source. The real challenge, consequently, is to ensure adequate fresh water supplies at the minimum possible cost. For these reasons, wind and wave energy coupled with desalination systems 
appear as a promising opportunity for small islands and developing countries to meet their water supply needs. Providing for these crucial needs via renewable natural resources, resulting in a broad social acceptance.

The water supply is not the only problem for Maldivian islands: the result of a study [74] shows that around $64 \%$ of all the islands reported severe beach erosion. Beach replenishment may only be a temporary remedy and traditional hard solutions, such as groynes, revetments or breakwaters, are often unpopular for aesthetic reasons and they reduce the recreational values. An interesting alternative may be represented by the beach drainage system, which has the great advantage to eliminate the visual impact. Additionally, its installation is not as costly as the traditional hard coastal structures and it does not suffer from wear rates as beach nourishments. This innovative system has been proven to stabilize the beachface when low incident wave energy conditions are ensured [75-77], like the ones inside a coral reef. In the context of the promotion of hybrid systems, it is remarkable that the drained water flows may be considered as a water pre-treatment for desalination, with both drainage and desalination plants powered by inshore/offshore blue energy technologies, pursuing a very high ethics perspective.

In order to boost blue growth, the paper investigates the uses of multifunctional structures combining power generation, desalinization and coastal defence. As aforementioned, two representative islands are analysed: Malé, and Magoodhoo. The selection of these sites provides two good case studies for different reasons. The first one is the opposite demographic conditions between the two islands. Then, the difference in the waterfront and coastal line, since Magoodhoo is a natural environment without any sign of significant human activity, while the Male Island is almost entirely protected by six-kilometers circumference of sea walls. Furthermore, the electricity demand in the capital island increases at a yearly rate of $11 \%$ [69] but, at the same time, there is not enough land to expand power plants. Therefore, it is crucial to find alternative options to supply electricity. Finally, Magoodhoo hosts the Marine Research and High Education Center born by the collaboration of Maldivian Government and the University of Milan-Bicocca. In particular, within the program "Benefits of using renewable energy for Maldivian communities and marine ecosystems" promoted with the support of the Italian Caritas, a photovoltaic system has been donated to the people of Magoodhoo [78]. This system feeds power to the island grid, and it is helping to reset the electricity production, moving from a small diesel sets configuration. Moreover, the cooperative agreement between Italy and the Maldives has also supported a new project termed "Installation of a water desalination plant on the island of Magoodhoo-Faafu Atoll" [79]. The desalination plant, through the use of solar thermal and photovoltaic panels, will provide clean water to the island's population, who currently does not have drinking water, and so to the MaRHE Center.

Nowadays, over 1000 wave energy converters (WECs) [80] have been patented over the last twenty years in order to transform wave energy into electrical energy. Several of these technologies are still in an early stage of technical development, and only few of them are ready for a pre-commercial stage (e.g., Aquabuoy, Aws II, Dexa wave, Limpet, Mutriku, OBREC, Oyster, Rewec III, Seabased, Pelamis, PowerBuoy, Wave Dragon, Wavestar, etc.) The selection of the best leading technologies or the tuning study of a specific WEC is out of the scope of this paper. Regarding the wave-to-wire efficiency of these technologies, from previous extensive literature [81-95] it is possible to assume, for a generic WEC, an overall averaged efficiency of $16.5 \%$. In order to cover the Malé electricity demand of $300 \mathrm{GWh} /$ year, a theoretical wave harvester over $20 \mathrm{~km}$ long should be required $(8.8 \mathrm{~kW} / \mathrm{m}$ are considered). Hence, wave energy seems more promising in supply energy in conjunction with other renewable sources. In particular, WECs have specific advantage for smaller islands, in order to maximize diesel savings in the short term and minimize the energy storage costs in the longer term. For instance, for Magoodhoo (where about $11.6 \mathrm{~kW} / \mathrm{m}$ are computed), just a ten meters wide WEC is able to supply the whole island electricity demand (approximately $150 \mathrm{MWh}$ ).

Wind resource in the Maldives is high in comparison to the energy demand. Tables 12 and 13 compare the theoretical wind power, $\mathrm{P}_{\text {wind }}$, the wind-to-wire power, $\mathrm{P}_{\text {tur }}$, and the annual averaged 
energy, $\mathrm{E}_{\text {yearly,mean, }}$ produced by the three aforementioned idealized WTGs at each Western and Eastern grid points. From an economic point of view, results show that larger turbines are more efficient. In fact, regarding the energy production, for one 2.3 MW WTG, three 1.0 MW WTGs and about 21 small $100 \mathrm{~kW}$ WTGs are needed. For a large turbine, the yearly energy could arise in average $4.33 \mathrm{GWh}$ on the west side and $5.11 \mathrm{GWh}$ on the eastern part of the archipelago. This represents an important result because about 100 wind generator of $2.3 \mathrm{MW}$ could provide the approximately $480 \mathrm{GWh}$ of the whole electricity sector, moving the Maldives towards energy self-sufficiency using offshore wind power.

However, such a high level of wind energy potential does not come without difficulties. First of all, the use of a single source of energy does not fit with energy security strategies, addressed to ensure the uninterrupted physical availability of energy products and to balance and diversify the various sources of supply. Secondly, visual impacts of onshore and nearshore wind facilities represent one on the major concern considering the high value of Maldive's natural seascapes. The environmental and socio-economic impact visual assets at highest risk from coastal and marine landscape change are directly linked to the height of wind towers and to the distance from the coastline [96]. Both the social acceptance of an offshore WTG and the economic appeal for investors and utility providers remain heavily dependent on costs vs. payback analysis, that means competitively priced electricity supplies and reliability.

A solution to significantly decrease the production costs of blue energies would be to develop hybrid technologies, i.e., co-locating floating/fixed WTGs and WECs at offshore locations, possibly by promoting multi-use platforms [13-23]. The combination of wind and wave energy resources reduce the overall dimensions per unit of power, the operational requirement for reserve and regulating power [97-101] as well as the requirement for generation capacity to maintain the power system reliability [102-104]. By the co-location of wind and wave generators, also the nearshore or shoreline devices are further promoted. Especially for the last case, devices embedded within coastal [93,105-109] or offshore infrastructures could be also combined with desalination plants. That solution has been recently considered in depth from many Authors (e.g., [110-113]). Numerous low-density population areas lack not only fresh water availability, but in most of the cases electrical grid connection or any other energy source as well [114]. Most of the countries experiencing "water stress" have enough water, but lack the means to provide it in an accessible manner [115]. For Magoodhoo, based on a specific energy consumption of $2.0 \mathrm{kWh} / \mathrm{m}^{3}$ [116] and water requirements of $1100 \mathrm{~m}^{3}$ per person per year (included domestic, drinking and food production purposes according to [117]), a $90 \mathrm{~m}$ wide WEC could ensure the total freshwater demand.

Moreover, this island has been recognized as one of the 86 islands that have reported severe beach erosion since 1990 [118]. In order to provide stabilization to the $2.3 \mathrm{~km}$ of Magoodhoo coastline with a beach drainage system (considered a low impact soft-engineering solution by coastal managers), a preliminary configuration has been assessed. For such a configuration, constituted by 4 parallel pipelines surrounding the island and pumping stations characterized by 12 pumps of $6.5 \mathrm{~kW}$ (adapted from [75] and [119]), an average annual electricity demand of about $200 \mathrm{MWh} € /$ year can be computed. Hence, a WEC $115 \mathrm{~m}$ wide could provide the total requests of energy, freshwater and beach stabilization purposes of the whole island. This proves the high benefits provided by WECs for small islands, according to $[56,120]$.

On the other hands, due to the lower wave resource in the eastern region, the about 114 million $\mathrm{m}^{3}$ /year of potable water for Malè requires large wind farms. A preliminary analysis using data for the 2.3 MW WTG, indicates as a number of 42 turbines are needed. Obviously, that number can reasonably be reduced if wind and wave harvesters are co-located. 
Table 12. Theoretical wind power, $\mathrm{P}_{\text {wind }}$, wind-to-wire power, $\mathrm{P}_{\text {turb }}$, and the annual averaged energy produced, $\mathrm{E}_{\text {yearly,mean }}$ by the three idealized WTGs at points W1-W5.

\begin{tabular}{|c|c|c|c|c|c|c|c|c|c|}
\hline Point & \multicolumn{3}{|c|}{$100 \mathrm{~kW}$ Turbine } & \multicolumn{3}{|c|}{ 1.0 MW Turbine } & \multicolumn{3}{|c|}{ 2.3 MW Turbine } \\
\hline W2 & 54.51 & 23.06 & 0.21 & 360.87 & 164.95 & 1.52 & 1247.81 & 485.50 & 4.52 \\
\hline W3 & 51.50 & 21.92 & 0.21 & 327.54 & 149.29 & 1.40 & 1179.00 & 459.01 & 4.32 \\
\hline W4 & 48.71 & 20.83 & 0.20 & 309.79 & 141.72 & 1.35 & 1115.08 & 435.09 & 4.16 \\
\hline
\end{tabular}

Table 13. Theoretical wind power, $\mathrm{P}_{\text {wind }}$, wind-to-wire power, $\mathrm{P}_{\text {turb }}$, and the annual averaged energy

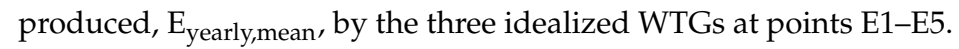

\begin{tabular}{|c|c|c|c|c|c|c|c|c|c|}
\hline Point & \multicolumn{3}{|c|}{$100 \mathrm{~kW}$ Turbine } & \multicolumn{3}{|c|}{ 1.0 MW Turbine } & \multicolumn{3}{|c|}{ 2.3 MW Turbine } \\
\hline E2 & 62.55 & 26.72 & 0.26 & 427.93 & 196.74 & 1.87 & 1432.00 & 571.09 & 5.45 \\
\hline E3 & 57.51 & 24.90 & 0.24 & 365.76 & 169.66 & 1.65 & 1316.56 & 525.39 & 5.11 \\
\hline E4 & 52.67 & 22.94 & 0.23 & 334.97 & 154.45 & 1.54 & 1205.73 & 482.29 & 4.76 \\
\hline
\end{tabular}

\section{Conclusions}

The present paper provides a preliminary assessment of the electric power generation of offshore wind turbines and wave energy converters along the Archipelago of the Maldives coasts. Wind and wave climate has been analysed and characterized using a 10-year period ECMWF wave forecasting data for 10 grid points. Both resources are relatively abundant, albeit opposite trends for energy potential have been recognized. While the wind power increases in the N-NE direction (moving from $72 \mathrm{~W} / \mathrm{m}^{2}$ to $104 \mathrm{~W} / \mathrm{m}^{2}$ in the study area), the average wave power density in deep waters ranges between $8.5 \mathrm{~kW} / \mathrm{m}$ in the north-eastern area to about $12.7 \mathrm{~kW} / \mathrm{m}$ in the south-west region. Furthermore, wind resource seems characterized by lower seasonal variability than wave energy. The energy patterns suggest that wind and waves are generally uncorrelated, especially during the period of southwest monsoon. These conditions are very interesting in the perspective of reducing the overall variability of the produced power.

This coastal area, therefore, shows to be an important candidate for the next optimal study site for several kinds of combined wind turbines and wave energy converters. Promising configuration of multi-use device is investigated through two case studies at Malè and Magoodhoo Island, in particular to face two main problems: water scarcity and beach erosion. Introducing additional considerations for environmental impact assessment, the high-density power provided by wave energy harvesters results as a good compromise for low-density population areas, lacking of not only fresh water availability, but in most of the cases also the electrical grid connection or any other energy source. However, very large offshore wind farms or co-located wind and wave farms are strongly recommended for higher energy demand areas. It has been demonstrated that renewable energy resources of offshore wind and wave can move the Maldives towards energy self-sufficiency. These combined farms would best be located near points along the coast where regions of high wind and wave resources overlap. The combined use of this resource, together with solar and biomass, has been shown to produce important benefits for the electric power system especially in the perspective of an energy diversification strategy.

Author Contributions: Pasquale Contestabile and Paolo Galli conceived and wrote the paper draft. Enrico Di Lauro performed the analysis for wave and wind energy assessment. Diego Vicinanza and Cesare Corselli revised the paper draft and Pasquale Contestabile updated the paper according to their review.

Conflicts of Interest: The authors declare no conflict of interest. 


\section{Abbreviations}

The following abbreviations are used in this manuscript:

$\begin{array}{ll}\text { ECMWF } & \text { European Centre for Medium-Range Weather } \\ \text { N-NE } & \text { North-NorthEast } \\ \text { S-SW } & \text { South-SouthWest } \\ \text { WEC } & \text { wave energy converter } \\ \text { WTG } & \text { wind turbine generator }\end{array}$

\section{Appendix A}

This appendix contains details about power production curves of the three practical utility-scale wind turbines analysed in this study.

As aforementioned the production curves are obtained averaging the ones provided by the following wind turbine manufacturers:

- $\quad$ for the $100 \mathrm{~kW}$ WTG, with hub height of $25 \mathrm{~m}$, the power curves of Northern Power System, Polaris America and Wind Energy Solutions are averaged;

for the 1.0 MW WTG, with nacelle height of $55 \mathrm{~m}$, the power curves of WTG proposed by WinWind and Nordic Windpower are considered;

finally, for the 2.3 MW WTG, with hub posed ad $80 \mathrm{~m}$, the production curves of the correspondent WTG size measured by General Electric and Siemens are averaged.

The resulting power curves are reported in Figure A1.
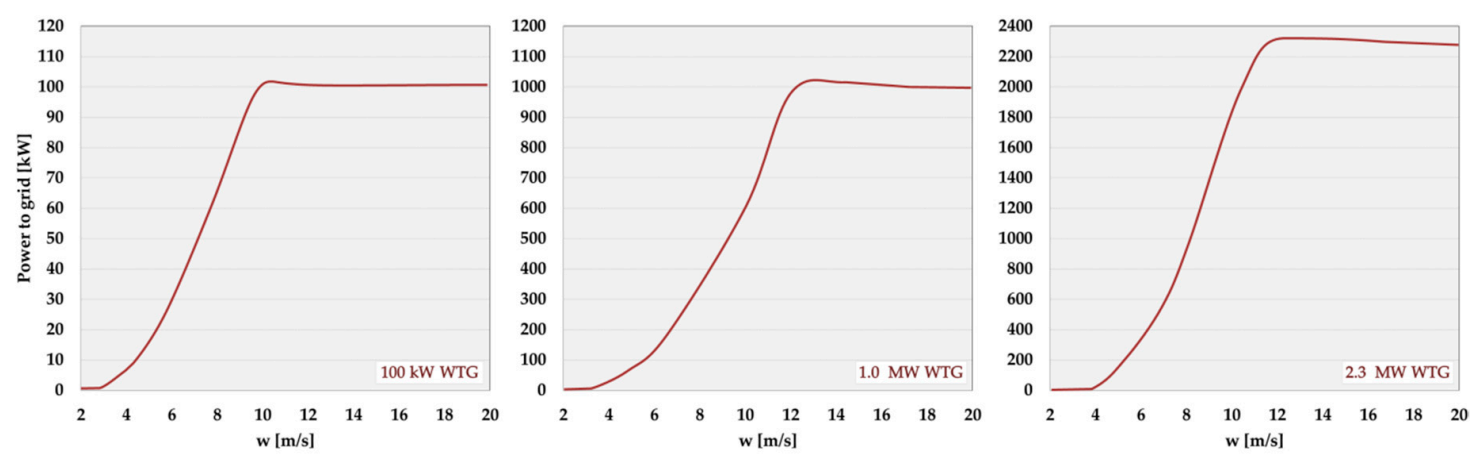

Figure A1. Power to grid vs. wind velocity at hub height (power curve) for the three wind turbines analysed.

\section{References}

1. Millar, J. The Ability of the Maldives to Cope with Freshwater Scarcity via the Adaptive Capacity of its Political Economy; Occasional Paper No. 44; Water Issues Study Group, School of Oriental and African Studies (SOAS), University of London: London, UK, 2002.

2. Ministry of Environment and Energy, Republic of the Maldive. Maldives SREP Investment plan 2013-2017. Available online: http://www.environment.gov.mv/v1/wp-content/blogs.dir/1/files/publications / 20131212-Pub-Maldives-SREP-Investment-Plan-2013-2017.pdf (accessed on 12 December 2016).

3. Kruijsen, J.H.J.; Joanneke, H.J.; Owen, A.; Turner, N. The potential of Marine Energy in the Maldives. In Proceedings of the World Congress on Water, Climate and Energy, Dublin, Ireland, 13-18 May 2012.

4. Renewable Energy Resource Mapping in the Maldives. Available online: https://www.esmap.org/re_ mapping_maldives (accessed on 12 February 2017).

5. Babarit, A.; Ben Ahmed, H.; Clément, A.H.; Debusschere, V.; Duclos, G.; Multon, B.; Robin, G. Simulation of electricity supply of an Atlantic island by offshore wind turbines and wave energy converters associated with a medium scale local energy storage. Renew. Energy 2006, 31, 153-160. [CrossRef]

6. Fusco, F.; Nolan, G.; Ringwood, J.V. Variability reduction through optimal combination of wind/wave resources-An Irish case study. Energy 2010, 35, 314-325. [CrossRef]

7. Lund, H. Large-scale integration of optimal combinations of $\mathrm{PV}$, wind and wave power into the electricity supply. Renew. Energy 2006, 31, 503-515. [CrossRef] 
8. Pontes, M.T.; Sempreviva, A.M.; Barthelmie, R.; Giebel, G.; Costa, P.; Sood, A. Integrating Offshore Wind and Wave Resource Assessment. Available online: https://www.researchgate.net/publication/228679581_ Integrating_Offshore_Wind_and_Wave_Resource_Assessment (accessed on 27 March 2017).

9. Veigas, M.; Iglesias, G. Wave and offshore wind potential for the island of Tenerife. Energy Convers. Manag. 2013, 76, 738-745. [CrossRef]

10. Azzellino, A.; Ferrante, V.; Kofoed, J.P.; Lanfredi, C.; Vicinanza, D. Optimal siting of offshore wind-power combined with wave energy through a marine spatial planning approach. Int. J. Mar. Energy 2013, 3, 11-25. [CrossRef]

11. Veigas, M.; Carballo, R.; Iglesias, G. Wave and offshore wind energy on an island. Energy Sustain. Dev. 2014, 22, 57-65. [CrossRef]

12. McAuliffe, F.D.; Macadré, L.; Donovan, M.H.; Murphy, J.; Lynch, K. Economic and Reliability Assessment of a Combined Marine Renewable Energy Platform. In Proceedings of the 11th European Wave and Tidal Energy Conference, Nantes, France, 6-11 September 2015.

13. Pérez-Collazo, C.; Greaves, D.; Iglesias, G. A review of combined wave and offshore wind energy. Renew. Sustain. Energy Rev. 2015, 42, 141-153. [CrossRef]

14. Astariz, S.; Perez-Collazo, C.; Abanades, J.; Iglesias, G. Towards the optimal design of a co-located wind-wave farm. Energy 2015, 84, 15-24. [CrossRef]

15. Abanades, J.; Greaves, D.; Iglesias, G. Coastal defence through wave farms. Coast. Eng. 2014, 91, $299-307$. [CrossRef]

16. Iglesias, G.; Carballo, R. Wave farm impact: The role of farm-to-coast distance. Renew. Energy 2014, 69, 375-385. [CrossRef]

17. Perez-Collazo, C.; Astariz, S.; Abanades, J.; Greaves, D.; Iglesias, G. Co-located wave and offshore wind farms: A preliminary case study of an hybrid array. In Proceedings of the 34th International Conference on Coastal Engineering, ICCE, Seoul, Korea, 15-20 June 2014.

18. Stoutenburg, E.D.; Jenkins, N.; Jacobson, M.Z. Power output variations of co-located offshore wind turbines and wave energy converters in California. Renew. Energy 2010, 35, 2781-2791. [CrossRef]

19. Veigas, M.; Iglesias, G. A hybrid wave-wind offshore farm for an Island. Int. J. Green Energy 2014, 12, 570-576. [CrossRef]

20. Fernandez Chozas, J.; Helstrup Jensen, N.E.; Sørensen, H.C. Economic benefit of combining wave and wind power productions in day-ahead electricity markets. In Proceedings of the 4 th International Conference on Ocean Energy, ICOE, Dublin, Ireland, 17-19 October 2012.

21. Fernandez Chozas, J.; Kofoed, J.P.; Kramer, M.M.; Sørensen, H.C. Combined production of a full-scale wave converter and a full-scale wind turbine e a real case study. In Proceedings of the 4th International Conference on Ocean Energy, ICOE, Dublin, Ireland, 17-19 October 2012.

22. Azzellino, A.; Contestabile, P.; Ferrante, V.; Lanfredi, C.; Vicinanza, D. Strategic environmental assessment to evaluate WEC projects in the perspective of the environmental cost-benefit analysis. In Proceedings of the International Offshore and Polar Engineering Conference, Maui, HI, USA, 19-24 June 2011; ISOPE: Maui, HI, USA, 2011; pp. 709-715.

23. Astariz, S.; Perez-Collazo, C.; Abanades, J.; Iglesias, G. Co-located wind-wave farm synergies (Operation \& Maintenance): A case study. Energy Convers. Manag. 2015, 91, 63-75.

24. World Wind Resource Assessment Report. Available online: http://www.wwindea.org/wp-content/ uploads/filebase/technology/WWEA_WWRAR_Dec2014_2.pdf (accessed on 27 March 2017).

25. Manwell, J.F.; Rogers, A.L.; McGowan, J.G.; Bailey, B.H. An offshore wind resource assessment study for New England. Renew. Energy 2002, 27, 175-187. [CrossRef]

26. Ucar, A.; Balo, F. Assessment of wind power potential for turbine installation in coastal areas of Turkey. Renew. Sustain. Energy Rev. 2010, 14, 1901-1912. [CrossRef]

27. Swart, R.J.; Coppens, C.; Gordijn, H.; Piek, M.; Ruyssenaars, P.; Schrander, J.J.; de Smet, P.; Hoogwijk, M.; Papalexandrou, M.; de Visser, E.; et al. Europe's Onshore and Offshore Wind Energy Potential: An Assessment of Environmental and Economic Constraints; European Environment Agency: Copenhagen, Denmark, 2009; Volume 6.

28. Bilgili, M.; Yasar, A.; Simsek, E. Offshore wind power development in Europe and its comparison with onshore counterpart. Renew. Sustain. Energy Rev. 2011, 15, 905-915. [CrossRef] 
29. Hasslet, J.; Kelledy, E. The assessment of actual wind power availability in Ireland. Int. J. Energy Res. 1997, 3, 333-348. [CrossRef]

30. Fagbenle, R.L.; Karayiannis, T.G. On the wind energy resource of Nigeria. Int. J. Energy Res. 1994, 18, 493-508. [CrossRef]

31. Celiktas, M.S.; Kocar, G. Foresight analysis of wind power in Turkey. Int. J. Energy Res. 2012, 36, 737-748. [CrossRef]

32. Veigas, M.; Iglesias, G. Evaluation of the wind resource and power performance of a turbine in Tenerife. J. Renew. Sustain. Energy 2012, 4, 053106. [CrossRef]

33. Vogiatzis, N.; Kotti, K.; Spanomitsios, S.; Stoukides, M. Analysis of wind potential and characteristics in North Aegean, Greece. Renew. Energy 2004, 29, 1193-1208. [CrossRef]

34. Rehman, S. Wind energy resources assessment for Yanbo, Saudi Arabia. Energy Convers. Manag. 2004, 45, 2019-2032. [CrossRef]

35. Li, M.; Li, X. Investigation of wind characteristics and assessment of wind energy potential for Waterloo region, Canada. Energy Convers. Manag. 2005, 46, 3014-3033. [CrossRef]

36. Ramachandra, T.V.; Shruthi, B.V. Wind energy potential mapping in Karnataka, India, using GIS. Energy Convers. Manag. 2005, 46, 1561-1578. [CrossRef]

37. Hasager, C.B.; Badger, M.; Pena, A.; Larse, X.G.; Bingol, F. SAR-based wind resource statistics in the Baltic Sea. Remote Sens. 2011, 3, 117-144. [CrossRef]

38. Alsaad, M.A. Wind energy potential in selected areas in Jordan. Energy Convers. Manag. 2013, 65, 704-708. [CrossRef]

39. Lizuma, L.; Avotniece, Z.; Rupainis, S.; Teilans, A. Assessment of the present and future offshore wind power potential: A case study in a target territory of the Baltic Sea near the Latvian Coast. Sci. World J. 2013, 52, 126482. [CrossRef] [PubMed]

40. Ubaldo, A. Wind Data Analysis and Wind Energy Potential in Italy. In International Conference on Environment and Renewable Energy. Available online: http://environment.scientific-journal.com/articles/ 3/27.pdf (accessed on 7 March 2017).

41. Mukasa, A.D.; Mutambatsere, E.; Arvanitis, Y.; Triki, T. Development of Wind Energy in Africa. Available online: https:/ / www.afdb.org/fileadmin/uploads/afdb/Documents/Publications/Working\%20Paper\% 20170\%20-\%20Development\%20of\%20Wind\%20Energy\%20in\%20Africa.pdf (accessed on 27 March 2017).

42. Ansari, M.Z.; Sharma, D.; Kumar, N.; Hossain, J. Offshore wind power development in India. Available online: http:/ / www.gwec.net/offshore-wind-development-in-india-fowind-launches-new-report-onsupply-chain-ports-and-logistics/ (accessed on 7 March 2017).

43. Lanfredi, N.W.; Pousa, J.L.; Mazio, C.A.; Dragani, W.C. Wave-power potential along the coast of the Province of Buenos Aires, Argentina. Energy 1992, 17, 997-1006. [CrossRef]

44. Nielsen, K.; Meyer, N.I. Danish Wave Energy Programme. In Proceedings of the 3rd European Wave Energy Conference, Patras, Greece, 30 September-2 October 1998.

45. Pontes, M.T. Assessing the European Wave Energy Resource. J. Offshore Mech. Arct. Eng. 1998, 120, $226-231$. [CrossRef]

46. Thorpe, T.W. The wave energy programme in the UK and the European wave energy network. In Proceedings of the 4th Wave Energy Conference, Aalborg, Denmark, 4-6 December 2000; pp. 19-27.

47. Clément, A.; McCullen, P.; Falcão, A.; Fiorentino, A.; Gardner, F.; Hammarlund, K.; Lemonis, G.; Lewis, T.; Nielsen, K.; Petroncini, S.; et al. Wave energy in Europe: Current status and perspectives. Renew. Sustain. Energy Rev. 2002, 6, 405-431. [CrossRef]

48. Folley, M.; Whittaker, T.J. Analysis of the nearshore wave energy resource. Renew. Energy 2009, 34, $1709-1715$. [CrossRef]

49. Iglesias, G.; Carballo, R. Wave energy potential along the Death Coast (Spain). Energy 2009, 34, $1963-1975$. [CrossRef]

50. Waters, R.; Engström, J.; Isberg, J.; Leijon, M. Wave climate off the Swedish west coast. Renew. Energy 2009, 34, 1600-1606. [CrossRef]

51. Mollison, D.; Pontes, M.T. Assessing the Portuguese wave-power resource. Energy 1992, 17, $255-268$. [CrossRef]

52. Iglesias, G.; Carballo, R. Wave energy and nearshore hot spots: The case of the SE Bay of Biscay. Renew. Energy 2010, 35, 2490-2500. [CrossRef] 
53. Chen, F.; Lu, S.M.; Tseng, K.T.; Lee, S.C.; Wang, E. Assessment of renewable energy reserves in Taiwan. Renew. Sustain. Energy Rev. 2010, 14, 2511-2528. [CrossRef]

54. Hughes, M.G.; Heap, A.D. National-scale wave energy resource assessment for Australia. Renew. Energy 2010, 35, 1783-1791. [CrossRef]

55. Abbaspour, M.; Rahimi, R. Iran atlas of offshore renewable energies. Renew. Energy 2011, 36, 388-398. [CrossRef]

56. Iglesias, G.; Carballo, R. Wave resource in El Hierro-an island towards energy self-sufficiency. Renew. Energy 2011, 36, 689-698. [CrossRef]

57. Kim, G.; Jeong, W.M.; Lee, K.S.; Jun, K.; Lee, M.E. Offshore and nearshore wave energy assessment around the Korean Peninsula. Energy 2011, 36, 1460-1469. [CrossRef]

58. Lenee-Bluhm, P.; Paasch, R.; Özkan-Haller, H.T. Characterizing the wave energy resource of the US Pacific Northwest. Renew. Energy 2011, 36, 2106-2119. [CrossRef]

59. Stopa, J.E.; Cheung, K.F.; Chen, Y.L. Assessment of wave energy resources in Hawaii. Renew. Energy 2011, 36, 554-567. [CrossRef]

60. Vicinanza, D.; Cappietti, L.; Ferrante, V.; Contestabile, P. Estimation of the wave energy in the Italian offshore. J. Coast. Res. 2011, 64, 613-617.

61. Vicinanza, D.; Contestabile, P.; Ferrante, V. Wave energy potential in the north-west of Sardinia (Italy). Renew. Energy 2013, 50, 506-521. [CrossRef]

62. Robertson, B.R.; Hiles, C.E.; Buckham, B.J. Characterizing the near shore wave energy resource on the west coast of Vancouver Island, Canada. Renew. Energy 2014, 71, 665-678. [CrossRef]

63. Iuppa, C.; Cavallaro, L.; Vicinanza, D.; Foti, E. Investigation of suitable sites for wave energy converters around Sicily (Italy). Ocean Sci. 2015, 11, 543-557. [CrossRef]

64. Jadidoleslam, N.; Özger, M.; Ağıralioğlu, N. Wave power potential assessment of Aegean Sea with an integrated 15-year data. Renew. Energy 2016, 86, 1045-1059. [CrossRef]

65. Contestabile, P.; Ferrante, V.; Vicinanza, D. Wave energy resource along the coast of Santa Catarina (Brazil). Energies 2015, 8, 14219-14243. [CrossRef]

66. Sierra, J.P.; Martín, C.; Mösso, C.; Mestres, M.; Jebbad, R. Wave energy potential along the Atlantic coast of Morocco. Renew. Energy 2016, 96, 20-32. [CrossRef]

67. Contestabile, P.; Di Lauro, E.; Buccino, M.; Vicinanza, D. Economic Assessment of Overtopping BReakwater for Energy Conversion (OBREC): A Case Study in Western Australia. Sustainability 2017, 9, 51. [CrossRef]

68. European Centre for Medium-RangeWeather Forecasts. Available online: http://www.ecmwf.int/ (accessed on 13 December 2016).

69. Maldives Energy Supply; Demand Survey 2010-2012. Available online: http://www.energy.gov.mv/v1/ wp-content/files/downloads/Maldives_Energy_Supply_Demand_Survey_2010_-_2012.pdf (accessed on 20 December 2016).

70. ABP Marine Environmental Research Ltd. Atlas of UK Marine Renewable Energy Resources: Technical Report; Report No. R.1106, Prepared for the UK Department of Trade and Industry; ABP Marine Environmental Research Ltd.: Southampton, UK, 2004.

71. Veritas, N. Environmental Conditions and Environmental Loads; Det Norske Veritas: Oslo, Norway, 2000.

72. Leroux, M. The Meteorology and Climate of Iropical Africa; Springer Science \& Business Medi: New York, NY, USA, 2001.

73. Holthuijsen, L.H.; Kuik, A.J.; Mosselman, E. The response of wave directions to changing wind directions. J. Phys. Oceanogr. 1987, 17, 845-853. [CrossRef]

74. Shaig, A. Climate change vulnerability and adaptation assessment of the land and beaches of maldives. In Technical Papers to Maldives National Adaptation Plan of Action for Climate Change; Ministry of Environment, Energy and Water: Amman, Jordan, 2006.

75. Contestabile, P.; Aristodemo, F.; Vicinanza, D.; Ciavola, P. Laboratory Study on a Beach Drainage System. Coast. Eng. 2012, 66, 50-64. [CrossRef]

76. Ciavola, P.; Vicinanza, D.; Aristodemo, F.; Contestabile, P. Large-scale morphodynamic experiments on a beach drainage system. J. Hydraul. Res. 2011, 49, 523-528. [CrossRef]

77. Ciavola, P.; Contestabile, P.; Aristodemo, F.; Vicinanza, D. Beach sediment mixing under drained and undrained conditions. J. Coast. Res. 2013, 65, 1503-1508. [CrossRef]

78. Renewable Energy Maldives. Available online: http://test.renewableenergymaldives.com.mv/universityof-milano-bicocca/ (accessed on 20 February 2017). 
79. Ministero Dell'ambiente e Della Tutela del Territorio e del Mare. Available online: http:/ /www.minambiente. it/pagina/maldive (accessed on 12 February 2017).

80. Antonio, F.D.O. Wave energy utilization: A review of the technologies. Renew. Sustain. Energy Rev. 2010, 14, 899-918.

81. De Andres, A.; Guanche, R.; Vidal, C.; Losada, I.J. Adaptability of a generic wave energy converter to different climate Conditions. Renew. Energy 2015, 78, 322-333. [CrossRef]

82. Zanuttigh, B.; Angelelli, E.; Kortenhaus, A.; Koca, K.; Krontira, Y.; Koundouri, P. A methodology for multi-criteria design of multi-use offshore platforms for marine renewable energy harvesting. Renew. Energy 2016, 85, 1271-1289. [CrossRef]

83. Koca, K.; Kortenhaus, A.; Oumeraci, H.; Zanuttigh, B.; Angelelli, E.; Cantu, M.; Suffredini, R.; Franceschi, G. Recent Advances in the Development of Wave Energy Converters. In Proceedings of the 10th European Wave and Tidal Energy Conference (EWTEC 2013), Aalborg, Denmark, 2-5 September 2013.

84. Taghipour, R. Efficient Prediction of Dynamic Response for Flexible and Multibody Marine Structures. Ph.D. Thesis, Faculty of Engineering Science and Technology, Norwegian University of Science and Technology, Trondheim, Norway, 2008.

85. Tello, M.; Bhattacharjee, J. Dynamics and hydrodynamics of a ship like Wave Energy Converters in roll. Marit. Eng. Technol. 2012. [CrossRef]

86. Guanche, R.; de Andres, A.; Simal, P.D.; Vidal, C.; Losada, I.J. Uncertainty analysis of wave energy farms finantial indicators. Renew. Energy 2013, 68, 570-580. [CrossRef]

87. da Ilurdoz Cortadellas, M.S.; Pereda, R.R.; Moreno, P.D.C. Preliminary Study for the Implementation of" Wave Dragon" on Isolated Spanish Networks with Subtropical Weather. J. Energy Power Eng. 2012, 6, 892-899.

88. Rusu, E. Evaluation of the wave energy conversion efficiency in various coastal environments. Energies 2014, 7, 4002-4018. [CrossRef]

89. Pecher, A. Performance Evaluation of Wave Energy Converters. Ph.D. Thesis, Department of Civil Engineering, Aalborg University, Aalborg, Denmark, 2012.

90. Meyer, N.I.; Arnskov, M.M.; Bennetzen, L.V.; Burcharth, H.F.; Bunger, J.; Jacobsen, V.; Maegaard, P.; Vindelov, S.; Nielsen, K.; Sørensen, J.N. Bølgekraftprogram: Afsluttende rapport fra Energistyrelsens Rådgivende Bølgekraftudvalg. Bølgekraftudvalgets Sekretariat Rambøll Teknikerbyen 2002, 31, 2830.

91. Pecher, A.; Kofoed, J.P.; Larsen, T.; Marchalot, T. Experimental Study of the WEPTOS Wave Energy Converter. In Proceedings of the 31th International Conference on Ocean, Offshore and Arctic Engineering, OMAE2012, Rio de Janeiro, Brazil, 1-6 July 2012.

92. Pecher, A.; Crom, I.L.; Kofoed, J.P.; Neumann, F.; Azevedo, E.D.B. Performance assessment of the Pico OWC power plant following the EquiMar methodology. In Proceedings of the 21th Internation Offshore (Ocean) and Polar Engineering Conference, ISOPE, Maui, HI, USA, 19-24 June 2011.

93. Torre-Enciso, Y.; Ortubia, I.; López de Aguileta, L.I.; Marqués, J. Mutriku wave power plant: From the thinking out to the reality. In Proceedings of the 8th European Wave and Tidal Energy Conference, Uppsala, Sweden, 7-10 September 2009; pp. 319-329.

94. Buccino, M.; Vicinanza, D.; Salerno, D.; Banfi, D.; Calabrese, M. Nature and magnitude of wave loadings at Seawave Slot-cone Generators. Ocean Eng. 2015, 95, 34-58. [CrossRef]

95. Vicinanza, D.; Nørgaard, J.H.; Contestabile, P.; Lykke-Andersen, T. Wave loadings acting on overtopping breakwater for energy conversion. J. Coast. Res. 2013, 65, 1669-1674. [CrossRef]

96. Azzellino, A.; Conley, D.; Vicinanza, D.; Kofoed, J. Marine Renewable Energies: Perspectives and Implications for Marine Ecosystems. Available online: https://www.hindawi.com/journals/tswj/2013/547563/ (accessed on 27 March 2017).

97. Milligan, M.; Porter, K.; Parsons, B.; Caldwell, J. Wind energy and power system operations: A survey of current research and regulatory actions. Electr. J. 2002, 15, 56-67. [CrossRef]

98. Parsons, B.K.; Wan, Y.; Kirby, B. Wind farm power fluctuations, ancillary services, and system operating impact analysis activities in the United States. In Proceedings of the European Wind Energy Conference, Copenhagen, Denmark, 2-6 July 2001.

99. Tipping, J. The Benefits of Marine Technologies within a Diversified Renewables Mix. Available online: http://www.parliament.scot/S4_EconomyEnergyandTourismCommittee/Inquiries/Pelamis_Wave. pdf (accessed on 7 March 2017). 
100. Holttinen, H. Impact of hourly wind power variations on the system operation in the Nordic countries. Wind Energy 2005, 8, 197-218. [CrossRef]

101. Holttinen, H.; Milligan, M.; Kirby, B.; Acker, T.; Neimane, V.; Molinski, T. Using standard deviation as a measure of increased operational reserve requirement for wind power. Wind Eng. 2008, 32, 355-377. [CrossRef]

102. Kahn, E. The reliability of distributed wind generators. Electr. Power Syst. Res. 1979, 2, 1-14. [CrossRef]

103. Milligan, M.; Artig, R. Reliability Benefits of Dispersed Wind Resource Development; National Renewable Energy Laboratory: Golden, CO, USA, 1998.

104. Wangdee, W.; Billinton, R. Considering load-carrying capability and wind speed correlation of WECS in generation adequacy assessment. IEEE Trans. Energy Convers. 2006, 21, 734-741. [CrossRef]

105. Contestabile, P.; Ferrante, V.; Di Lauro, E.; Vicinanza, D. Prototype overtopping breakwater for wave energy conversion at port of Naples. In Proceedings of the Twenty-Sixth International Ocean and Polar Engineering Conference, Rhodes, Greece, 26 June-1 July 2016; pp. 616-621.

106. Contestabile, P.; Iuppa, C.; Di Lauro, E.; Cavallaro, L.; Lykke Andersen, T.; Vicinanza, D. Wave loadings acting on innovative rubble mound breakwater for overtopping wave energy conversion. Coast. Eng. 2017, 122, 60-74. [CrossRef]

107. Vicinanza, D.; Contestabile, P.; Nørgaard, J.; Lykke-Andersen, T. Innovative rubble mound breakwaters for overtopping wave energy conversion. Coast. Eng. 2014, 88, 154-170. [CrossRef]

108. Buccino, M.; Stagonas, D.; Vicinanza, D. Development of a composite sea wall wave energy converter system. Renew. Energy 2015, 81, 509-522. [CrossRef]

109. Arena, F.; Romolo, A.; Malara, G.; Ascanelli, A. On design and building of a U-OWC wave energy converter in the Mediterranean Sea: A case study. In Proceedings of the 32nd International Conference on Ocean, Offshore and Arctic Engineering, Nantes, France, 9-14 June 2013.

110. Garcia-Rodriguez, L. Renewable energy applications in desalination: State of the art. Sol. Energy 2003, 75, 381-393. [CrossRef]

111. Hicks, D.C.; Mitcheson, G.R.; Pleass, C.M.; Salevan, J.F. Delbuoy: Ocean wave-powered seawater reverse osmosis desalination systems. Desalination 1989, 73, 81-94. [CrossRef]

112. Tsiourtis, N.X. Desalination and the environment. Desalination 2001, 141, 223-236. [CrossRef]

113. Kalogirou, S.A. Seawater desalination using renewable energy sources. Prog. Energy Combust. Sci. 2005, 31, 242-281. [CrossRef]

114. Mathioulakis, E.; Belessiotis, V.; Delyannis, E. Desalination by using alternative energy: Review and state-of-the-art. Desalination 2007, 203, 346-365. [CrossRef]

115. Sharmila, N.; Jalihal, P.; Swamy, A.K.; Ravindran, M. Wave powered desalination system. Energy 2004, 29, 1659-1672. [CrossRef]

116. Folley, M.; Whittaker, T. The cost of water from an autonomous wave-powered desalination plant. Renew. Energy 2009, 34, 75-81. [CrossRef]

117. Allan, J.A.; Allan, T. The Middle East Water Question Hydropolitics and the Global Economy; I.B.Tauris: London, UK, 2001.

118. National Bureau of Statistics-Ministry of Finance and Treasury (Maldives). Available online: http: //www.planning.gov.mv/publications/yrb2004/yrb04/Yearbook/2.Environment/2.2.htm/ (accessed on 13 December 2016).

119. Ciavola, P.; Vicinanza, D.; Fontana, E. Beach drainage as a form of shoreline stabilization: Case studies in Italy. In Proceedings of the 31st International Conference on Coastal Engineering, Hamburg, Germany, 10-25 April 2009; pp. 2646-2658.

120. Sandberg, A.B.; Klementsen, E.; Muller, G.; de Andres, A.; Maillet, J. Critical Factors Influencing Viability of Wave Energy Converters in Off-Grid Luxury Resorts and Small Utilities. Sustainability 2016, 8, 1274. [CrossRef]

(c) 2017 by the authors. Licensee MDPI, Basel, Switzerland. This article is an open access article distributed under the terms and conditions of the Creative Commons Attribution (CC BY) license (http:/ / creativecommons.org/licenses/by/4.0/). 